Pacific

Journal of

Mathematics

FOLIATIONS ON SUPERMANIFOLDS

AND CHARACTERISTIC CLASSES

Camille Laurent-GengouX

Volume $228 \quad$ No. 1

November 2006 


\title{
FOLIATIONS ON SUPERMANIFOLDS AND CHARACTERISTIC CLASSES
}

\author{
CAmille Laurent-GengouX
}

\begin{abstract}
We construct secondary characteristic classes of regular superfoliations on smooth supermanifolds. We interpret these secondary characteristic classes as characteristic classes of flat foliated connections.
\end{abstract}

\section{Introduction}

Smooth supermanifolds are becoming an increasingly important subject in mathematical physics [Leites 1980]; superfoliations should then be a central object. In this paper, we construct secondary characteristic classes of regular superfoliations with trivialized normal bundle, in the spirit of [Bernstein and Rosenfeld 1973; Bott and Haefliger 1972; Fuchs 1986]. We show that the role of Vect $(n)$ (the Lie algebra of formal vector fields with $n$ variables) from the classical theory is played by the even part $\operatorname{Vect}(n, m)_{0}$ of the super-Lie algebra $\operatorname{Vect}(n, m)$ of formal supervector fields with $n$ even variables and $m$ odd variables. More precisely, when on a supermanifold $M$ we are given a superfoliation $\mathscr{F}$ of codimension $n+\varepsilon m$ with trivialized normal bundle, we will associate to any element $H$ in the Chevalley-Eilenberg cohomology $H^{*}\left(\operatorname{Vect}(n, m)_{0}\right)$ a secondary characteristic class $\varphi_{\mathcal{M}, \mathscr{F}}(H) \in H^{*}(M)$, where $M$ is the base manifold of the supermanifold $M$.

Theorem A. For any supermanifold $M$ foliated by a superfoliation $\mathscr{F}$ of codimension $n+\varepsilon m$ with trivialized normal bundle, there exists a natural homomorphism $\varphi_{\mathcal{M}, \mathscr{F}}$ from $H^{*}\left(\operatorname{Vect}(n, m)_{0}\right)$ to $H^{*}(M)$ such that:

(1) If $\Phi: \mathcal{N} \rightarrow \mathcal{M}$ is a submersion of supermanifolds and $\varphi: N \rightarrow M$ is the map induced by $\Phi$ on their base manifolds $M$ and $N$, then

$$
\varphi_{\mathcal{N}, \Phi * \mathscr{F}}=\varphi^{*} \circ \varphi_{\mathcal{M}, \mathscr{F}},
$$

where $\Phi^{*} \mathscr{F}_{\text {F }}$ is the pull-back of the superfoliation $\mathscr{F}$ via $\Phi$ and $\varphi^{*}: H^{*}(M) \rightarrow$ $H^{*}(N)$ is the pull-back through $\varphi$.

MSC2000: primary 14M30; secondary 53C10.

Keywords: superfoliation, supergeometry, secondary characteristic classes. 
(2) If $\mathcal{M}$ is an ordinary smooth manifold endowed with a foliation $F$ of codimension $n$ with trivialized normal bundle, then $\varphi_{F}$ reduces to the well-known homomorphism of Bernstein, Bott, Fuchs, Haefliger and Rosenfeld [Bernstein and Rosenfeld 1973; Bott and Haefliger 1972; Fuchs 1973].

The reader should not be surprised that the de Rham cohomology of $\mu$ is not used: by a result of Batchelor [Leites 1980], this cohomology is indeed isomorphic to the cohomology of the base manifold. Note that the even part Vect $(n, m)_{0}$ appears instead of $\operatorname{Vect}(n, m)$ itself. The theory is indeed more interesting like this, since, for example, $H^{*}(\operatorname{Vect}(n, m))$ has only two nontrivial generators for $n<m$ (see [Fuchs 1973]). In [Koszul 1988], Godbillon-Vey classes for superfoliations of codimension $0+\varepsilon m$ are constructed on supermanifolds of superdimension $n+\varepsilon m$ as maps from $H^{*}(\operatorname{Vect}(0, m))$ to $H^{*}(\mathcal{M})$. We will see that these classes vanish for any class $H \in H^{*}(\operatorname{Vect}(0, m))$ whose restriction to the cohomology $H^{*}\left(\operatorname{Vect}(0, m)_{0}\right)$ of the even part is 0 . Furthermore, we will show in Section 4D that the classes constructed in [Koszul 1988] are among those built in this article.

Given a vector bundle $E \rightarrow M$ and a foliation $F$, a foliated connection means a connection on $E$ defined (smoothly) over each leaf of the foliation $F$. A foliated connection is said to be flat if it is flat on each leaf, and trivial if $E$ is a trivial vector bundle and $F$ a foliation with trivialized normal bundle. For simplicity, we say flat trivial foliated connection to name the entire collection of a foliation with trivialized normal bundle, a vector bundle, and a flat foliated connection. There is a canonical way to construct, from a flat foliated connection, a superfoliation with trivialized normal bundle on a supermanifold. Therefore, our theory of secondary characteristic classes of superfoliations also gives a theory of secondary characteristic classes of flat foliated connections.

Furthermore, to a superfoliation $\mathscr{F}$ of codimension $n+\varepsilon m$ with trivialized normal bundle on a supermanifold $\mathcal{M}$ we shall associate a flat trivial foliated connection, that is, a foliation $F_{\mathscr{F}}$ of codimension $n$ with trivialized normal bundle on the base manifold $M$, a trivial vector bundle $E_{\mathscr{F}}$ of dimension $m$ over $M$, and a flat foliated connection $\nabla^{\mathscr{F}}$ of this bundle. It should be noted that this construction is not the inverse of the previous one. However, we shall see that our theory of secondary characteristic classes, because of our preceding construction, gives in fact a theory of secondary characteristic classes of flat foliated connections. More precisely, we show:

Theorem B. The homomorphism $\varphi_{\mathscr{F}, M}: H^{*}\left(\operatorname{Vect}(n, m)_{0}\right) \rightarrow H^{*}(M)$ is completely determined by the flat trivial foliated connection $\left(M, F_{\mathscr{F}}, E_{\mathscr{F}}, \nabla^{\mathscr{F}}\right)$.

The paper is organized as follows. Section 2 is devoted to constructions and properties of superfoliations on supermanifolds. More precisely, in Section 2A 
we introduce several useful maps; in Section 2B we recall the definition of a superfoliation; in Section $2 \mathrm{C}$ we exhibit relations between superfoliations and flat foliated connections; in Section 2D we show how to replace a superfoliation with a superfoliation with trivialized normal bundle.

Section 3 is devoted to the study of the Chevalley-Eilenberg cohomology of the Lie algebra $\operatorname{Vect}(n, m)_{0}$. We introduce this cohomology in Section $3 \mathrm{~A}$, then present in Section 3B technical results that will play a fundamental role in the construction of secondary characteristic classes. We show in Section 3C how to compute the cohomology of $\operatorname{Vect}(n, m)_{0}$ with the help of the Weil algebra.

We construct secondary characteristic classes of superfoliations with trivialized normal bundle in Section 4. Secondary characteristic classes of a superfoliation are defined in Section 4A by constructing a homomorphism of differential graded algebras from the complex $\bigwedge \operatorname{Vect}(n, m)_{0}$ of the Lie algebra $\operatorname{Vect}(n, m)_{0}$ to the de Rham complex $\Omega(M)$ of the manifold $M$. We give examples in Section $4 \mathrm{~B}$, and prove Theorem A (in fact, a more precise statement) in Section 4D.

We relate in Section 5A the previously constructed secondary characteristic classes to the secondary characteristic classes of the (ordinary) foliation $F_{\mathscr{F}}$ on the base manifold. We prove Theorem B in Section 5B.

Most result of this paper have been announced, but not proved, in [LaurentGengoux 2004].

\section{Superfoliations}

2A. The algebra of superdifferential forms. For any supervector space $\mathscr{V}$, denote by $\mathscr{V}_{0}$ its even part. For convenience, write DGA for "differential graded algebra". For a DGA $\left(A, d_{A}\right)$, denote by $[a] \in H^{*}(A)$ the cohomology class of an element $a \in \operatorname{Ker} d_{A}$. Throughout this article, $M$ is a smooth supermanifold of superdimension $p+\varepsilon q$ ( $p$ and $q$ being nonnegative integers), and $M$ is its $p$-dimensional base manifold. In the sequel, we say "dimension" for "superdimension", and "codimension" for "supercodimension"

We now recall some basic results about supermanifolds, and introduce some maps that we will need in the sequel.

Denote by $O(M)$ the superalgebra of superfunctions on $\mathcal{M}$, and by $\mathscr{I}(\mathcal{M})$ the superalgebra of nilpotent superfunctions; denote by $\operatorname{Re}(f)$ the smooth function on $M$ defined by the canonical projection $O(M) \rightarrow \mathcal{O}(M) / \mathscr{I}(\mathcal{M}) \simeq \mathscr{C}^{\infty}(M, \mathbb{R})$.

Let $(\Omega(\mathcal{M}), \wedge)$ be the superalgebra of differential forms on $\mathcal{M}$. The notion of parity of a superdifferential form can be ambiguous; in this paper the parity is the one that endows the superalgebra $\Omega(\mathcal{M})$ with a structure of a supercommutative superalgebra. More precisely, in a local chart $\left(x_{1}, \ldots, x_{p}, \theta_{1}, \ldots, \theta_{q}\right)$, the 1 -form $d x_{i}$ is odd, the 1 -form $d \theta_{j}$ is even, the 0 -form $x_{i}$ is even, and the 0 -form $\theta_{j}$ is odd. 
The parity is then extended by multiplication: for any $\omega_{1}, \omega_{2} \in \Omega(\mathcal{M})$, the parity of $\omega_{1} \wedge \omega_{2}$ is the sum of the parity of $\omega_{1}$ with the parity of $\omega_{2}$ (modulo 2). For example, $\theta_{j} d x_{i}$ is even, $x_{i}^{2} d \theta_{j}$ is even, and $d x_{i} \wedge d \theta_{j}$ is odd. Note that, with this definition, a 1-form on an ordinary differential manifold is an odd element. Let $d_{\mathcal{M}}: \Omega^{*}(\mathcal{M}) \rightarrow \Omega^{*+1}(\mathcal{M})$ be the de Rham differential.

Let $E_{\mathcal{M}}$ be a $q$-dimensional vector bundle over $M$ whose sheaf of sections is $\mathscr{I}(M) / \mathscr{I}(M)^{2}$. The supermanifold $M(M, E)$ whose superfunctions are the sections of $\wedge^{*} E_{\mathcal{M}}$ is isomorphic to $\mathcal{M}$ (as a supermanifold, see [Leites 1980]). A local chart $\left(x_{1}, \ldots, x_{p}, \theta_{1}, \ldots, \theta_{q}\right)$ is said to be attached to $E_{\mathcal{M}}$ if the odd superfunctions $\theta_{1}, \ldots, \theta_{q}$ are identified with sections $s_{1}, \ldots, s_{q}$ of $E_{\mathcal{M}}$. Note that in this case the supervector fields $\partial / \partial \theta_{1}, \ldots, \partial / \partial \theta_{q}$ can be identified with sections of the dual vector bundle $E_{\mathcal{M}}^{*}$, and the 1 -forms $d \theta_{1}, \ldots, d \theta_{q}$ can be identified with sections of $E_{\mathcal{M}}$ again. More precisely, there is a natural bijection $\mathscr{Y}$ from the vector bundle whose sections are 1 -forms in $\Omega^{1}(\mathcal{M})$ of the form $\sum_{j=1}^{q} f_{j}\left(x_{1}, \ldots, x_{p}\right) d \theta_{j}$ to the vector bundle $E_{\mathcal{M}}$ given, in a local chart attached to $E_{\mathcal{M}}$, by

$$
\mathscr{S}\left(\sum_{j=1}^{q} f_{j}\left(x_{1}, \ldots, x_{p}\right) d \theta_{j}\right)=\sum_{j=1}^{q} f_{j}\left(x_{1}, \ldots, x_{p}\right) s_{j},
$$

with $f_{1}\left(x_{1}, \ldots, x_{p}\right), \ldots, f_{q}\left(x_{1}, \ldots, x_{p}\right)$ smooth functions on $M$. It is straightforward to check that $\mathscr{S}$ does not depend on the local chart attached to $E_{\mathcal{M}}$.

We recall the definition of three maps:

- The first map is a well-known (see [Leites 1980; Tuynman 2004]) canonical DGA homomorphism $p$ from $\left(\Omega^{*}(\mathcal{M}), \wedge, d_{M}\right)$ onto $\left(\Omega^{*}(M), \wedge, d_{M}\right)$, constructed in [Leites 1980]. In a local chart $\left(x_{1}, \ldots, x_{p}, \theta_{1}, \ldots, \theta_{q}\right)$, it is defined on the generators $d x_{i}, d \theta_{j}, f$ ( $f$ being a superfunction) of $\Omega(\mathcal{M})$ by

$$
p\left(d x_{i}\right)=d x_{i}, \quad p\left(d \theta_{i}\right)=0, \quad p(f)=\operatorname{Re}(f) .
$$

A proof of the next lemma can be found in [Leites 1980; Tuynman 2004].

Lemma 2.1. The map p from $\left(\Omega^{*}(\mathcal{M}), \wedge, d_{M}\right)$ onto $\left(\Omega^{*}(M), \wedge, d_{M}\right)$ is well defined (that is, independent of the chart) and a DGA homomorphism. The kernel of $p$ contains odd $2 k$-forms and even $(2 k+1)$-forms.

Since $p$ is a DGA homomorphism, it induces a homomorphism $\hat{p}$ from $H^{*}(\mathcal{M})$ to $H^{*}(M)$. According to a theorem of Batchelor (see [Tuynman 2004, Theorem 8.2]), $\hat{p}: H^{*}(\mathcal{M}) \rightarrow H^{*}(M)$ is indeed an isomorphism.

- The second map, $\rho$, is a map from $\Omega^{1}(\mathcal{M})$ to $\Gamma\left(E_{\mathcal{M}}\right)$, the space of sections of $E_{\mathcal{M}}$. It is defined in a local chart by

$$
\rho\left(\sum_{i=1}^{p} f_{i} d x_{i}+\sum_{j=1}^{q} g_{i} d \theta_{i}\right)=\mathscr{S}\left(\sum_{j=1}^{q} \operatorname{Re}\left(g_{j}\right) d \theta_{j}\right)=\sum_{j=1}^{q} \operatorname{Re}\left(g_{j}\right) s_{j} .
$$


Lemma 2.2. The map $\rho$ is well defined (that is, it does not depend on the chart attached to $\left.E_{\mathcal{M}}\right)$. For any odd 1-form $\omega$, we have $\rho(\omega)=0$. For any nilpotent function $f \in \mathscr{I}(\mathcal{M})$ and any $\omega \in \Omega^{1}(M)$, we have $\rho(f \omega)=0$.

- The third map that we need, $\Pi$, is a map from the super-Lie algebra $\operatorname{Vect}(\mathcal{M})$ of supervector fields on $\mathcal{M}$ to the Lie algebra $\operatorname{Vect}(M)$ of vector fields on $M$. For any odd supervector field $\mathscr{L} \in \operatorname{Vect}(\mathcal{M})$, we simply set $\Pi(\mathscr{L})=0$. For any even supervector field $\mathscr{X} \in \operatorname{Vect}(\mathcal{M})_{0}$, we set $\Pi(\mathscr{X})=X$, where $X$ is the derivation of $C^{\infty}(M, \mathbb{R}) \simeq \mathscr{O}(\mathcal{M}) / \mathscr{I}(\mathcal{M})$ induced by the even derivation $\mathscr{X}$ of $\mathcal{O}(\mathcal{M})$.

In a local chart attached to $E_{\mathcal{M}}, \Pi$ is given by

$$
\Pi\left(\sum_{i=1}^{p} f_{i} \frac{\partial}{\partial x_{i}}+\sum_{j=1}^{q} g_{j} \frac{\partial}{\partial x_{j}}\right)=\sum_{i=1}^{p} \operatorname{Re}\left(f_{i}\right) \frac{\partial}{\partial x_{i}} .
$$

Lemma 2.3. The restriction of $\Pi$ to the subalgebra $\operatorname{Vect}(\mathcal{M})_{0}$ of even supervector fields is a Lie algebra homomorphism.

The next properties can be checked locally in a chart; we leave the computations to the reader. Denote by $\iota_{\text {oy }} \eta$ the contraction of a supervector field by a 1 -form in $\Omega(M)$. For any 2 -form $\eta \in \Omega^{2}(\mathcal{M})$ and even supervector field $y$ such that $\Pi(\mathscr{y})=0$,

$$
\rho\left(\iota_{\mathrm{g}} \eta\right)=0 .
$$

For any even 1-form $\alpha \in \Omega^{1}(\mathcal{M})$ with $\rho(\alpha)=0$ and any even supervector field $\mathscr{Y}$,

$$
\rho\left(\iota_{\text {gy }} d \alpha\right)=0 \text {. }
$$

For any even supervector field $\mathscr{L} \in \operatorname{Vect}(\mathcal{M})_{0}$ and odd 1 -form $\omega \in \Omega^{1}(\mathcal{M})$,

$$
\operatorname{Re}\left(\iota_{\mathscr{L}} \omega\right)=\iota_{\Pi(X)} p(\omega) .
$$

2B. Definition of a superfoliation. We recall the definition of a superfoliation of codimension $n+\varepsilon m$ [Leites 1980; Monterde et al. 1997; Tuynman 2004]. First, a distribution of codimension $n+\varepsilon m$ is a sub-supervector bundle of $T M$ of dimension $(p-n)+\varepsilon(q-m)$.

Definition 2.4. A superfoliation $\mathscr{F}$ of codimension $n+\varepsilon m$ is a distribution $\mathscr{D}_{\mathscr{F}}$ of dimension $(p-n)+\varepsilon(q-m)$ whose sections are closed under the bracket of supervector fields.

Remark 2.5. Note that in the literature the terminology is not fixed: in [Leites 1980] the name "foliation" is used, while in [Tuynman 2004] it is called "integrable distribution". We choose "superfoliation" to avoid confusions when dealing simultaneously with superfoliations on a supermanifold and (ordinary) foliations on its base manifold. 
A supermanifold is said to be foliated when it is endowed with a superfoliation of codimension $n+\varepsilon m$, for some $n, m \in \mathbb{N}$. A supervector field $\mathscr{X}$ is said to be tangent to the leaves if and only if it is a section of the distribution that defines $\mathscr{F}$. We denote by $\mathscr{X}_{\mathscr{F}_{F}}$ the superalgebra of supervector fields tangent to the leaves of $\mathscr{F}$.

For a superfoliation $\mathscr{F}_{\mathscr{F}}$, denote by $\Omega_{\mathscr{F}}^{*}$ the subalgebra of $\Omega(\mathcal{M})$ of $k$-forms $\omega$ such that $\iota_{\mathscr{O}_{1}} \ldots \iota_{\mathscr{O}_{k}} \omega=0$ for any supervector fields $\mathscr{Y}_{1}, \ldots, \mathscr{Y}_{k}$ tangent to the leaves of $\mathscr{F}$. Of course,

$$
d_{M} \Omega_{\mathscr{F}}^{*} \subset \Omega_{\mathscr{F}}^{*} .
$$

In particular, $\Omega_{\mathscr{F}}^{1}$ is the space of 1 -forms $\omega \in \Omega^{1}(\mathcal{M})$ such that $\omega(\mathscr{X})=0$ for any supervector field $\mathscr{X}$ tangent to the leaves.

A superfunction is said to be constant on the leaves if $\iota_{\mathscr{X}} d_{\mathcal{M}} f=0$ for any supervector field $\mathscr{X}$ tangent to the leaves. We denote by $O_{\mathscr{F}}$ the superalgebra of superfunctions constant on the leaves of $\mathscr{F}$.

Here is a basic example: On the supermanifold $\mathbb{R}^{p, q}$, denote by $x_{1}, \ldots, x_{p}$ (respectively, $\theta_{1}, \ldots, \theta_{q}$ ) the even (respectively, odd) variables. The elementary superfoliation $\mathbb{R}_{n, m}^{p, q}$ is the superfoliation given by the distribution generated by the supervector fields $\partial / \partial x_{n+1}, \ldots, \partial / \partial x_{p}, \partial / \partial \theta_{m+1}, \ldots, \partial / \partial \theta_{q}$.

An important particular case is when the superfoliation is defined by a distribution that admits a trivialized normal bundle.

Definition 2.6. A superfoliation $\mathscr{F}$ of codimension $n+\varepsilon m$ is said to have a trivialized normal bundle if the distribution $\mathscr{D}_{\mathscr{F}}$ that defines $\mathscr{F}_{\mathscr{F}}$ is generated by a free family of $n$ odd and $m$ even 1 -forms $b_{1}, \ldots, b_{n+m}$; that is,

$$
\mathscr{D}_{\mathscr{F}}=\left\{\mathscr{X} \in \operatorname{Vect}(M) \mid \iota_{\mathscr{X}} b_{1}=\cdots=\iota_{\mathscr{X}} b_{n+m}=0\right\} .
$$

We say that the 1 -forms $b_{1}, \ldots, b_{n+m}$ define the superfoliation $\mathscr{F}$.

Example 2.7. The elementary superfoliation $\mathbb{R}_{n, m}^{p, q}$ is defined by the odd 1-forms $d x_{1}, \ldots, d x_{n}$ together with the even 1 -forms $d \theta_{1}, \ldots, d \theta_{m}$.

If the superfoliation $\mathscr{F}_{F}$ with trivialized normal bundle is defined by a free family $b_{1}, \ldots, b_{n+m}$, then (see [Monterde et al. 1997]) there must exist 1 -forms $b_{i}^{j}, i, j \in$ $\{1, \ldots, n+m\}$, satisfying

$$
d_{M} b_{i}=\sum_{i=1}^{n+m} b_{j} \wedge b_{i}^{j} .
$$

Moreover, we can assume that the parity of $b_{i}^{j}$ is the sum of the parity of $b_{i}$ with the parity of $b_{j}$. 
Remark 2.8. For any superfoliation $\mathscr{F}_{F}$ with trivialized normal bundle, defined by 1 -forms $b_{1}, \ldots, b_{n+m}$ (with $b_{1}, \ldots, b_{n}$ odd, and $b_{n+1}, \ldots, b_{n+m}$ even), there exist supervector fields $X_{1}, \ldots, X_{n+m}$ (with $X_{1}, \ldots, X_{n}$ even, and $X_{n+1}, \ldots, X_{n+m}$ odd) such that $b_{i}\left(X_{j}\right)=\delta_{i}^{j}$ (where $\delta_{i}^{j}$ is the Kronecker symbol). The family $X_{1}, \ldots, X_{n+m}$ is free (with respect to the $\mathcal{O}(\mathcal{M})$-module structure). The supertangent bundle $T M$ is the direct sum of the distribution generated by $X_{1}, \ldots, X_{n+m}$ with the distribution $\mathscr{D}_{\mathscr{F}}$ that defines $\mathscr{F}$. This justifies calling it "with trivialized normal bundle".

We now define morphisms between foliated supermanifolds, and pull-backs of superfoliations.

Definition 2.9. Let $\mu_{1}$ and $\mu_{2}$ be supermanifolds, and let $\mathscr{F}_{1}$ and $\mathscr{F}_{2}$ be superfoliations of codimension $n+\varepsilon m$ on $M_{1}$ and $\mu_{2}$ respectively. A submersion $\Phi: \mathcal{M}_{1} \rightarrow M_{2}$ is said to be a morphism of superfoliations if and only if

$$
\mathcal{O}\left(M_{1}\right) \Phi^{*} \Omega_{\mathscr{F}_{2}}^{1}=\Omega_{\mathscr{F}_{1}}^{1},
$$

where $\mathcal{O}\left(\mu_{1}\right) \Phi^{*} \Omega_{\mathscr{F}_{2}}^{1}$ denotes the $\mathcal{O}\left(M_{1}\right)$-module generated by $\Phi^{*} \Omega_{\mathscr{F}_{2}}^{1}$

In other words, (2-7) means that any 1-form in $\Omega_{\mathscr{F}_{1}}$ is a linear combination, with coefficients in $O\left(M_{1}\right)$, of pull-backs by $\Phi$ of forms from $\Omega_{\mathscr{F}_{2}}$.

Note that, if $\mathscr{F}_{1}$ has a trivialized normal bundle, so has $\mathscr{F}_{2}$. If $b_{1}, \ldots, b_{n+m}$ are the 1 -forms defining $\mathscr{F}_{1}$, then the superfoliation $\mathscr{F}_{2}$ is defined by the 1 -forms $\Phi^{*} b_{1}, \ldots, \Phi^{*} b_{n+m}$.

The category of foliated supermanifolds is the category whose objects are superfoliations and whose arrows are morphism of foliated manifolds. For any $n, m \in \mathbb{N}$, the superfoliations of codimension $n+\varepsilon m$ make up a subcategory, while the superfoliations of codimension $n+\varepsilon m$ with trivialized normal bundle form again a subcategory of the latter category.

An isomorphism of foliated manifolds is an invertible morphism of foliated manifolds.

The local structure of a superfoliation is always the same, as proved in [Hill and Simanca 1991] (see also [Tuynman 2004, Chapter V.4]):

Theorem 2.10 [Hill and Simanca 1991; Monterde et al. 1997]. Any superfoliation of codimension $n+\varepsilon m$ on a supermanifold of dimension $p+\varepsilon q$ is locally isomorphic to the elementary superfoliation $\mathbb{R}_{n, m}^{p, q}$.

Let $\mathcal{N}$ and $\mathcal{M}$ be supermanifolds, and let $\mathscr{F}$ be a superfoliation of codimension $n+\varepsilon m$ on $\mathcal{M}$. Given any submersion $\Phi: \mathcal{N} \rightarrow \mathcal{M}$, the pull-back $\Phi^{*} \mathscr{F}_{F}$ is the unique superfoliation satisfying

$$
\mathcal{O}(\mathcal{N}) \Phi^{*} \Omega_{\mathscr{F}}^{1}=\Omega_{\Phi * \mathscr{F}}^{1} .
$$


This definition needs some justification. For any open set $U \subset M$, we denote by $\mathcal{U}$ the supermanifold defined by $\left.\mathcal{O}(\mathcal{M})\right|_{U}$ and we say that $\mathcal{U}$ is an open subset of $M$. Let $r+\varepsilon s$, with $r \geq p$ and $s \geq q$, be the dimension of $\mathcal{N}$. By Theorem 2.10, in local coordinates $\left(x_{1}, \ldots, x_{p}, \theta_{1}, \ldots, \theta_{q}\right)$ the superfoliation $\mathscr{F}$ restricted to $\mathcal{U}$ is defined by $d x_{1}, \ldots, d x_{n}, d \theta_{1}, \ldots, d \theta_{m}$. Since $\Phi$ is a submersion, there are local coordinates $\left(x_{1}, \ldots, x_{r}, \theta_{1}, \ldots, \theta_{s}\right)$ on some open subset $\mathscr{V} \subset \mathcal{N}$ such that $\Phi$ is given by

$$
\Phi\left(x_{1}, \ldots, x_{r}, \theta_{1}, \ldots, \theta_{s}\right)=\left(x_{1}, \ldots, x_{p}, \theta_{1}, \ldots, \theta_{q}\right) .
$$

The pull-back $\Phi^{*} \mathscr{F}_{F}$ of $\mathscr{F}$ via $\Phi$ is the superfoliation whose restriction to $\mathscr{V}$ is defined by the 1 -forms $d x_{1}, \ldots, d x_{n}, d \theta_{1}, \ldots, d \theta_{m} \in \Omega^{1}(\mathscr{V})$. This justifies the existence and uniqueness of the pull-back of a superfoliation via a submersion.

2C. Geometric constructions associated to a superfoliation. Let $N$ be an (ordinary) smooth manifold and $F$ a foliation of codimension $n$ on $N$, defined by a distribution $D_{F}$. Let $E \rightarrow N$ be a vector bundle over $N$, and let $X_{F}=\Gamma\left(D_{F}\right)$ be the algebra of vector fields tangent to $F$. A foliated connection is a bilinear map from $X_{F} \otimes \Gamma(E)$ to $\Gamma(E)$, denoted by $(X, s) \mapsto \nabla_{X} s$, that satisfies the usual axioms of a connection; that is, for all $X \in X_{F}, f \in C^{\infty}(M), s \in \Gamma(E)$, we have

$$
\nabla_{f X} s=f \nabla_{X} s \quad \text { and } \quad \nabla_{X} f s=f \nabla_{X} s+(X \cdot f) s .
$$

A foliated connection is said to be flat if $\nabla$ is flat on each leaf of $F$; that is, if for all $X, Y \in X_{F}, s \in \Gamma(E)$ the identity

$$
\nabla_{X} \nabla_{Y} s-\nabla_{Y} \nabla_{X} s-\nabla_{[X, Y]} s=0
$$

holds. A foliated connection is said to be trivial if $E$ is a trivial vector bundle on $M$ and $F$ is a foliation with trivialized normal bundle on $M$.

In the following, we will say simply "foliated connection" (respectively, "trivial flat foliated connection") for the collection of a foliation $F$, a vector bundle $E$, and a foliated connection $\nabla$. We denote by $(M, F, E, \nabla)$ a foliated connection (respectively, a trivial flat foliated connection).

2C(a). From superfoliations to flat foliated connections. To a superfoliation on $M$ we associate a foliation $F_{\mathscr{F}}$ on the base manifold $M$, a vector bundle $E_{\mathscr{F}}$ on $M$, and a flat foliated connection $\nabla^{\mathscr{F}}$ on the latter. In the particular case of a superfoliation of codimension $0+\varepsilon m$ on a supermanifold of dimension $n+\varepsilon m$, this construction is identical to the construction given in [Koszul 1988, Lemme 2.1].

Definition 2.11. Given a superfoliation $\mathscr{F}$ of codimension $n+\varepsilon m$, we denote by $\left(M, F_{\mathscr{F}}, E_{\mathscr{F}}, \nabla^{\mathscr{F}}\right)$ the flat foliated connection defined in what follows.

It is easy to check that $p\left(\Omega_{\mathbb{R}_{n, m}^{p, q}}^{*}\right)=\Omega_{\mathbb{R}_{n}^{p}}^{*}$, where by $\mathbb{R}_{n}^{p}$ we mean the foliation $\mathbb{R}_{n, 0}^{p, 0}$ of codimension $n$ on $\mathbb{R}^{p}$. In other words, $\mathbb{R}_{n}^{p}$ is the foliation of codimension $n$ on $\mathbb{R}^{p}$ defined by the 1 -forms $d x_{n+1}, \ldots, d x_{p}$. Theorem 2.10 immediately implies: 
Lemma 2.12. The distribution whose sections are $X_{F_{\mathscr{F F}}}=\Pi\left(\mathscr{X}_{\mathscr{F}_{F}}\right) \subset \Gamma(T M)$ defines a foliation $F_{\mathscr{F}}$ of codimension $n$ on $M$. Moreover, $\Omega_{F_{F}}^{*}=p\left(\Omega_{\mathscr{F}}^{*}\right)$.

Remark 2.13. If $\mathscr{F}$ is a superfoliation with trivialized normal bundle, defined by $1-$ forms $\omega_{1}, \ldots, \omega_{n}, a_{1}, \ldots, a_{m}$, then $F_{\mathscr{F}}$ is a foliation with trivialized normal bundle defined by the 1 -forms $p\left(\omega_{1}\right), \ldots, p\left(\omega_{n}\right) \in \Omega^{1}(M)$.

Now, we define $E_{\mathscr{F}}$ to be the vector subbundle of $E_{\mathcal{M}}$ whose sections $\Gamma\left(E_{\mathscr{F F}}\right)$ are the sections of $E_{\mathcal{M}}$ of the form $\rho(\omega)$ with $\omega \in \Omega_{\mathscr{F}}^{1}$, where $\Omega_{\mathscr{F}}^{1}$ is the space of 1-forms such that $\iota_{\mathscr{L}} \omega=0$ for any supervector field $\mathscr{X} \in \mathscr{X}_{\mathscr{F}}$ tangent to the leaves of $\mathscr{F}_{F}$, and $\rho$ was defined in (2-1). In the case of $\mathbb{R}_{n, m}^{p, q}, E_{\mathscr{F}}$ is generated by $\mathscr{S}\left(d_{\mathbb{R}^{p, q}} \theta_{1}\right), \ldots, \mathscr{Y}\left(d_{\mathbb{R}^{p, q}} \theta_{m}\right)$. Then, Theorem 2.10 implies that $E_{\mathscr{F}}$ is an $m$-dimensional vector bundle on $M$.

Remark 2.14. If $\mathscr{F}$ is a superfoliation with trivialized normal bundle, defined by 1 forms $\omega_{1}, \ldots, \omega_{n}, a_{1}, \ldots, a_{m}$, then $E_{\mathscr{F}}$ is a trivial vector space, and a trivialization is given by the sections $\rho\left(a_{1}\right), \ldots, \rho\left(a_{m}\right) \in \Gamma\left(E_{\mathscr{F}}\right)$.

For any section $s$ of $E_{\mathscr{F}}$, let $\omega \in \Omega_{\mathscr{F}}^{1}$ be an even element satisfying $\rho(\omega)=s$. For any vector field $Y \in X_{F_{\mathscr{F}}}$ tangent to the foliation $F_{\mathscr{F}}$, let $\mathscr{Y} \in\left(\mathscr{X}_{\mathscr{F}}\right)_{0}$ be an even supervector field satisfying $\Pi(Y)=Y$. We define a foliated connection by

$$
\nabla_{Y}^{\mathscr{F}} s=\rho\left(L_{\text {oy }} \omega\right) .
$$

Lemma 2.15. The foliated connection $\nabla_{Y}^{\mathscr{F}} s$ is well defined and flat.

Proof. Since $L_{\mathscr{y}}=d_{M} \iota_{\text {○y }}+\iota_{\circlearrowleft y} d_{\mu}$, the identity

$$
\rho\left(L_{\text {○ु }} \omega\right)=\rho\left(\iota_{\text {○y }} d_{\mathcal{M}} \omega\right)
$$

holds. In order to check that $\nabla_{Y}^{\mathscr{F}} s=\rho\left(L_{\mathscr{O}} \omega\right)$ is a well-defined section of $E_{\mathscr{F}}$, notice these facts:

- Let $\mathscr{L} \in \mathscr{X}_{\mathscr{F}}$ be a supervector field tangent to the leaves of $\mathscr{F}$ such that $\Pi(\mathscr{L})=$ 0 . Equation (2-2) implies that $\rho\left(L_{\mathscr{L}} \omega\right)=\rho\left(\iota_{\mathscr{L}} d_{\mathcal{M}} \omega\right)$ is equal to 0 .

- Let $\alpha \in \Omega_{\mathscr{F}}^{1}$ be an even 1-form such that $\rho(\alpha)=0$. Equation (2-3) implies that $\rho\left(L_{Y} \alpha\right)=0$.

We now verify that indeed $\nabla^{\mathscr{F}}$ is a section of $E_{\mathscr{F}}$. If $\omega \in \Omega_{\mathscr{F}}^{1}$, then $L_{\mathscr{y}} \omega$ is an element of $\Omega_{\mathscr{F}}^{1}$ as well, since

$$
\iota_{\mathscr{X}} L_{\mathscr{y}} \omega=\iota_{[\mathscr{X}, \mathscr{Y}]} \omega-\iota_{\mathscr{y}} L_{\mathscr{X}} \omega=0
$$

for all $X \in \mathscr{X}_{\mathscr{F}}$. The section $\nabla_{Y}^{\mathscr{F}} s$ of $E_{\mathcal{M}}$ given by $\nabla_{Y}^{\mathscr{F}} s=\rho\left(L_{\mathscr{O}} \omega\right)$ is thus a section of $E_{\mathscr{F}}$ again.

Now, we check that the curvature is zero. For this, we show that there exists a local trivialization of $E_{\mathscr{F}}$ by sections $s_{1}, \ldots, s_{m}$ that are horizontal (that is, $\nabla_{Y}^{\mathscr{F}} s_{i}=0$ 
for all $\left.Y \in X_{F_{F}}\right)$. For any $x \in M$, let $U$ be a neighborhood of $x$ and $\varphi_{U}: U \rightarrow$ $\mathbb{R}_{n, m}^{p, q}$ an isomorphism of foliated manifolds, where $U$ is the supermanifold over $U$ defined by restricting $\mathcal{M}$ to $U$. Since the superfunctions $\theta_{1}, \ldots, \theta_{m}$ are constant on the leaves of $\mathbb{R}_{p, q}^{n, m}$, the odd superfunctions $f_{1}=\varphi_{U}^{*} \theta_{1}, \ldots, f_{m}=\varphi_{U}^{*} \theta_{m} \in \Omega(\mathcal{M})$ are constant on the leaves of $\mathscr{F}$. Moreover, the sections $s_{1}=\rho\left(d_{\mathcal{M}} f_{1}\right), \ldots, s_{n}=\rho\left(d_{\mathcal{M}} f_{m}\right)$ define a local trivialization of $E_{\mathscr{F}}$. For any vector field $Y \in X_{F_{F}}$ and any even supervector field $\mathscr{Y} \in\left(\mathscr{X}_{\mathscr{F}}\right)_{0}$ with $\Pi(\mathscr{Y})=Y$, we have

$$
\nabla_{Y}^{\mathscr{F}} s_{i}=\rho\left(L_{\odot y} d_{M} f_{i}\right)=\rho\left(d_{M} \iota_{\circlearrowleft} d_{M} f_{i}\right)=\rho(0)=0 .
$$

The existence of a local horizontal trivialization of $E_{\mathscr{F}}$ implies that the connection $\nabla^{\mathscr{F}}$ is flat.

We summarize:

Proposition 2.16. Let $\mathscr{F}$ be a superfoliation of codimension $n+\varepsilon m$.

(1) $\left(M, F_{\mathscr{F}}, E_{\mathscr{F}}, \nabla^{\mathscr{F}}\right)$ is a flat foliated connection.

(2) If the superfoliation $\mathscr{F}_{F}$ has a trivialized normal bundle, then the flat foliated connection $\left(M, F_{\mathscr{F}}, E_{\mathscr{F}}, \nabla^{\mathscr{F}}\right)$ is trivial.

Proof. Statement (1) is a consequence of Lemma 2.12, while (2) is a consequence of Remarks 2.13 and 2.14

2C(b). From flat foliated connections to superfoliations. Let $(N, F, E, \nabla)$ be a flat foliated connection. Consider the supermanifold $\mathcal{M}(N, E)$ whose superfunctions are the sections of $\bigwedge E$. For any vector field $X \in \operatorname{Vect}(N), \nabla_{X}$ is an even derivation of the superalgebra of superfunctions $\Gamma(\bigwedge E)$. These even derivations can be considered as even vector fields of the supermanifold $\mathcal{M}(N, E)$. Moreover, since $\left[\nabla_{X}, \nabla_{Y}\right]=\nabla_{[X, Y]}$, these even derivations form a Lie algebra. The $\Gamma(\bigwedge E)$ module generated by the supervector fields $\left\{\nabla_{X} \mid X \in X_{F}\right\}$ is a distribution that defines a superfoliation of codimension $n+\varepsilon m$ on the supermanifold $M(N, E)$.

This provides a canonical way to associate a superfoliation of codimension $n+\varepsilon m$ to a manifold $N$, a foliation $F$ of codimension $n$, a vector-bundle $E$ of dimension $m$, and a flat foliated connection $\nabla$.

Definition 2.17. We denote by $\mathscr{F}(N, F, E, \nabla)$ the superfoliation of codimension $n+\varepsilon m$ on the supermanifold $\mathcal{M}(N, E)$, defined above.

A foliated connection $(N, F, E, \nabla)$ is said to be trivial if $F$ has a trivialized normal bundle and the vector bundle $E$ is trivial. The next proposition is straightforward and we leave it to the reader.

Proposition 2.18. (1) If $(N, F, E, \nabla)$ is a trivial flat foliated connection, then the superfoliation $\mathscr{F}(N, F, E, \nabla)$ has a trivialized normal bundle. 
(2) For any foliated connection $(M, F, E, \nabla)$, we have

$$
(M, F, E, \nabla)=\left(M, F_{\mathscr{F}(M, F, E, \nabla)}, E_{\mathscr{F}(M, F, E, \nabla)}, \nabla^{\mathscr{F}(M, F, E, \nabla)}\right) .
$$

Remark 2.19. By (2-9), the construction of Section $2 \mathrm{C}$ (a) is the left inverse of the construction of Section 2C(b). However, it is not a right inverse. More precisely, we show now that the construction of Section $2 \mathrm{C}$ (a) is not injective.

Consider the supercircle $S^{1,3}$ defined by a trivial vector bundle $E_{3}=S^{1} \times \mathbb{R}^{3} \rightarrow$ $S^{1}$ of dimension 3 over $S^{1}$. Denote by $x \in S^{1}$ the even parameter and by $\theta_{1}, \theta_{2}, \theta_{3}$ the odd parameters associated to three canonical sections $s_{1}, s_{2}, s_{3}$ of $E_{3}=S^{1} \times$ $\mathbb{R}^{3} \rightarrow S^{1}$.

Take the superfoliations $\mathscr{F}_{1}$ and $\mathscr{F}_{2}$ defined by the distributions of codimension $0+\varepsilon 3$ generated by the distributions $\mathcal{O}(\mathcal{M}) \partial / \partial x$ and $\mathcal{O}(\mathcal{M})\left(\partial / \partial x+\theta_{1} \theta_{2} \theta_{3} \partial / \partial \theta_{1}\right)$. These two superfoliations define the same foliation on $M$, the foliation with only one leaf: $S^{1}$ itself. They define the same vector bundle: $E_{3}$ itself. They both also define the same connection, namely, the connection given by

$$
\nabla_{a(x) \frac{\partial}{\partial x}} s=a(x) \frac{d s}{d x}
$$

for all $a(x) \in C^{\infty}\left(S^{1}\right)$ and $s \in \Gamma\left(E_{3}\right)$. However, these superfoliations are not isomorphic, because their superalgebras of superfunctions constant on leaves are not isomorphic.

For the superfoliation $\mathscr{F}_{1}$, the superfunctions $\theta_{1}, \theta_{2}, \theta_{3}$ are constant on leaves, and the algebra of superfunctions that are constant on the leaves of $\mathscr{F}_{1}$ is therefore isomorphic to $\bigwedge \mathbb{R}^{3}$. In particular, the vector space of odd superfunctions that are constant on the leaves of $\mathscr{F}_{1}$ has dimension 4.

For the superfoliation $\mathscr{F}_{2}$, any odd superfunction $f$ can be written

$$
f=\sum_{i=1}^{3} f_{i}(x) \theta_{i}+g(x) \theta_{1} \theta_{2} \theta_{3}
$$

with $f_{1}, f_{2}, f_{3}, g \in \mathscr{C}^{\infty}\left(S^{1}, \mathbb{R}\right)$. If $f$ is an odd superfunction constant on the leaves of $\mathscr{F}_{2}$, then

$$
\left(\frac{\partial}{\partial x}+\theta_{1} \theta_{2} \theta_{3} \frac{\partial}{\partial \theta_{1}}\right) \cdot f=0 \quad \text { and } \quad \sum_{i=1}^{3} \frac{d f_{i}(x)}{d x} \theta_{i}+\left(\frac{d g(x)}{d x}+f_{1}(x)\right) \theta_{1} \theta_{2} \theta_{3}=0 .
$$

This implies that $d f_{1} / d x=d f_{2} / d x=d f_{3} / d x=0$ and $d g / d x+f_{1}(x)=0$. Hence, for any $i \in\{1,2,3\}$, we obtain $f_{i}(x)=a_{i}$ for some constant $a_{i} \in \mathbb{R}$. But the equation $d g / d x+a_{1}=0$ has no periodic solution unless $a_{1}=0$. As a consequence, the vector space of odd superfunctions that are constant on the leaves of $\mathscr{F}_{2}$ has only dimension 3. The superfoliations $\mathscr{F}_{1}$ and $\mathscr{F}_{2}$ are therefore not isomorphic. 
2D. Superfoliations with trivialized normal bundle. We describe a canonical process to replace a superfoliation on $M$ by a superfoliation with trivialized normal bundle on a $G L(n, m)$-bundle $\mathscr{P}_{\mathscr{F}}$ over $M$. For a definition of the super-Lie group $G L(n, m)$, see [Leites 1980; Tuynman 2004].

The tangent bundle of the superfoliation $\mathscr{F}$ is a supervector subbundle of $T M$, of dimension $(p-n)+\varepsilon(q-m)$. The normal bundle $\mathscr{G}$ of $\mathscr{F}$ is a supervector subbundle of $T^{*} \mathcal{M}$ of dimension $n+\varepsilon m$. Let $\mathscr{P}_{\mathscr{F}}$ be the frame bundle of $\varphi_{\text {; }}$ this is a $G L(n, m)$-bundle over $\mathcal{M}$. Denote by $\pi: \mathscr{P}_{\mathscr{F}} \rightarrow \mathcal{M}$ the canonical projection on $M$.

By the construction of a frame bundle, there is a canonical inclusion of $\mathscr{P}_{\mathscr{F}}$ into $\mathscr{G}+\mathscr{G}[1] \subset\left(T^{*} \mathcal{M}\right)^{\oplus n} \oplus T^{*} \mathcal{M}[1]^{\oplus m}$, where $\mathscr{G}[1]$ and $T^{*} \mathcal{M}[1]$ are the supervector bundles obtained by reversing parities on the fibers. Let $\alpha$ be the canonical odd 1 -form of $T^{*} M$ and $\beta$ the canonical even 1-form of $T^{*} M[1]$. Since we have not been able to locate these 1-forms in the existing literature, we introduce them now. Consider local coordinates on $T^{*} \mu$

$$
\left(x_{1}, \ldots, x_{p}, \theta_{1}, \ldots, \theta_{q}, y_{1}, \ldots, y_{p}, \eta_{1}, \ldots, \eta_{q}\right) \text {, }
$$

where $x_{1}, \ldots, x_{p}, \theta_{1}, \ldots, \theta_{q}$ are local coordinates on $\mu$ and $y_{1}, \ldots, y_{p}, \eta_{1}, \ldots, \eta_{q}$ are the even and odd coordinates on the cotangent bundle of $\mu$ corresponding to the basis dual to $\partial / \partial x_{1}, \ldots, \partial / \partial x_{p}, \partial / \partial \theta_{1}, \ldots, \partial / \partial \theta_{q}$ of $T M$. We define $\alpha$ as

$$
\alpha=\sum_{i=1}^{p} y_{i} d x_{i}-\sum_{j=1}^{p} \eta_{j} d \theta_{j}
$$

It is routine to check that $\alpha$ does not depend on the local coordinates $x_{1}, \ldots, x_{p}$, $\theta_{1}, \ldots, \theta_{q}$ on $\mu$. The 1 -form $\beta$ is defined by the same formula, where $y_{1}, \ldots, y_{p}$ are now considered to be odd variables and $\eta_{1}, \ldots, \eta_{q}$ are considered to be even variables.

For $i=1, \ldots, n$, denote by $f_{i}: \mathscr{P}_{\mathscr{F}} \rightarrow T^{*} \mathcal{M}$ the $i$-th projection on $T^{*} \mathcal{M}$ and, for $j=1, \ldots, m$, denote by $g_{j}: \mathscr{P}_{\mathscr{F}_{F}} \rightarrow T^{*} M$ the $j$-th projection on $T^{*} \mathcal{M}[1]$. Define a family of 1 -forms on $\mathscr{P}_{\mathscr{F}}$ as

$$
\omega_{i}=f_{i}^{*} \alpha, \quad i=1, \ldots, n, \quad \text { and } \quad a_{j}=g_{j}^{*} \beta, \quad j=1, \ldots, m .
$$

Proposition 2.20. The pull-back $\pi^{*} \mathscr{F}$ of the superfoliation $\mathscr{F}$ by $\pi$ is a superfoliation on $\mathscr{P}_{\mathscr{F}}$ of codimension $n+\varepsilon m$ with trivialized normal bundle, defined by the 1 -forms $\omega_{1}, \ldots, \omega_{n}, a_{1}, \ldots, a_{m}$.

Proof. According to Theorem 2.10, we just have to check that Proposition 2.20 holds in the case of the superfoliation $\mathbb{R}_{n, m}^{p, q}$. Let $\left(x_{1}, \ldots, x_{p}, \theta_{1}, \ldots, \theta_{q}\right)$ be the (global) coordinates of $\mathbb{R}_{n, m}^{p, q}$ and $y_{i}^{j}, \eta_{k}^{j}, \zeta_{i}^{l}, z_{k}^{l}$, for $i, j \in\{1, \ldots, n\}$ and $k, l \in$ $\{1, \ldots, m\}$, and with $\operatorname{det}\left(y_{i}^{k}\right) \neq 0$ and $\operatorname{det}\left(z_{k}^{l}\right) \neq 0$, be the (global) coordinates of $G L(n, m)$. These coordinates define a system of coordinates on the supermanifold 
$\mathscr{P}_{\mathbb{R}_{n, m}^{p, q}} \simeq \mathbb{R}_{n, m}^{p, q} \times G L(n, m)$. In the latter system, we have

$$
\omega_{i}=\sum_{k=1}^{n} y_{k}^{i} d x_{k}-\sum_{l=1}^{m} \theta_{l}^{i} d \theta_{l} \quad \text { and } \quad a_{j}=\sum_{k=1}^{n} \zeta_{k}^{j} d x_{k}-\sum_{l=1}^{m} z_{l}^{j} d \theta_{l} .
$$

Since $\operatorname{det}\left(y_{i}^{k}\right) \neq 0$ and $\operatorname{det}\left(z_{k}^{l}\right) \neq 0$, the 1 -forms $\omega_{1}, \ldots, \omega_{n}, a_{1}, \ldots, a_{m} \in \Omega^{1}\left(\mathscr{P}_{\mathbb{R}_{n, m}^{p, q}}\right)$ and the 1 -forms $d x_{1}, \ldots, d x_{n}, d \theta_{1}, \ldots, d \theta_{m} \in \Omega^{1}\left(\mathscr{P}_{\mathbb{R}_{n, m}^{p, q}}\right)$ define the same superfoliation on $\mathscr{P}_{\mathbb{R}_{n, m}^{p, q}}$. This superfoliation is clearly $\pi^{*} \mathscr{F}_{\mathscr{F}}$. This completes the proof.

Remark 2.21. Proposition 2.20 provides a canonical way to replace a superfoliation $\mathscr{F}$, which has no trivialized normal bundle, by a superfoliation $\pi^{*} \mathscr{F}_{F}$ that has a trivialized normal bundle. This construction is functorial, in the sense that, if $\mathscr{F}_{1}$ is a superfoliation on $\mu_{1}$, then $\mathscr{F}_{2}$ is a superfoliation on $M_{2}$, and, if $\Phi$ : $\mu_{1} \rightarrow M_{2}$ is a morphism of foliated supermanifolds, then $\Phi$ induces a submersion $\Phi_{\mathscr{P}}: \mathscr{P}_{\mathscr{F}_{1}} \rightarrow \mathscr{P}_{\mathscr{F}_{2}}$ that is a morphism of foliated supermanifolds with respect to the superfoliations $\pi^{*} \mathscr{F}_{1}$ and $\pi^{*} \mathscr{F}_{2}$. In other words, the application $\mathscr{F}_{F} \mapsto \mathscr{P}_{\mathscr{F}}$ defines a functor from the category of foliated supermanifolds of codimension $n+\varepsilon m$ to the category of foliated supermanifolds of codimension $n+\varepsilon m$ with trivialized normal bundle.

\section{The cohomology of $\operatorname{Vect}(n, m)_{0}$}

For any super-Lie algebra $\mathfrak{g}$, let $X_{1}, \ldots, X_{g}$ be a basis of $\mathfrak{g}$ and $a_{1}, \ldots, a_{g}$ the dual basis, with $a_{i}\left(X_{i}\right)=(-1)^{\left|X_{i}\right|}$, where $\left|X_{i}\right|$ is the parity of $X_{i}$, and $a_{i}\left(X_{j}\right)=0$ for $i \neq j$. If for any $i, j, k \in\{1, \ldots, g\}$ there exists some $C_{i, j}^{k} \in \mathbb{R}$ with

$$
\left[X_{i}, X_{j}\right]=\sum_{k=1}^{g} C_{i, j}^{k} X_{k},
$$

then the Chevalley-Eilenberg differential is given by (see [Fuchs 1986])

$$
\delta a_{k}=\frac{1}{2} \sum_{i, j=1}^{g}(-1)^{\left|X_{i}\right|} C_{i, j}^{k} a_{i} \wedge a_{j} .
$$

Since we work with infinite-dimensional Lie algebras, we have to replace the Chevalley-Eilenberg cohomology by an infinite-dimensional generalization, but the signs in Equation (3-1) will remain valid.

3A. The Chevalley-Eilenberg complex of $\operatorname{Vect}(n, m)$ and $\operatorname{Vect}(n, m)_{0}$. To construct the theory of secondary characteristic classes of superfoliations, we need some prerequisites about the cohomology of a certain infinite-dimensional Lie algebra of supervector fields; this construction can be found, for instance, in [Fuchs 1986]. 
Take the superalgebra of superpolynomials

$$
\mathbb{R}\left[x_{1}, \ldots, x_{n}, \theta_{1}, \ldots, \theta_{m}\right]=\mathbb{R}\left[x_{1}, \ldots, x_{n}\right] \otimes \wedge \mathbb{R}^{m} .
$$

The degree of a superpolynomial $x_{1}^{i_{1}} \ldots x_{n}^{i_{n}} \theta_{1}^{j_{1}} \ldots \theta_{m}^{j_{m}}$, with $i_{1}, \ldots, i_{n} \in \mathbb{N}$ and $j_{1}, \ldots, j_{m} \in\{0,1\}$, is defined to be $i_{1}+\cdots+i_{n}+j_{1}+\cdots+j_{m}$. This turns $\mathbb{R}\left[x_{1}, \ldots, x_{n}, \theta_{1}, \ldots, \theta_{m}\right]$ into an $\mathbb{N}$-graded superalgebra.

The graded super-Lie algebra

$$
\operatorname{Vect}(n, m)=\bigoplus_{i=-1}^{+\infty} \operatorname{Vect}(n, m)^{i}
$$

of formal supervector fields is defined to be the super-Lie algebra of superderivations of $\mathbb{R}\left[x_{1}, \ldots, x_{n}, \theta_{1}, \ldots, \theta_{m}\right]$. Elements of $\operatorname{Vect}(n, m)^{i}$ are said to be of weight $i$. We denote $\operatorname{Vect}(n, 0)$ simply by $\operatorname{Vect}(n)$. We denote the even part of $\operatorname{Vect}(n, m)$ by $\operatorname{Vect}(n, m)_{0}=\bigoplus_{i=-1}^{+\infty} \operatorname{Vect}(n, m)_{0}^{i}$.

Let $\wedge \operatorname{Vect}(n, m)^{*}$ be the DGA of multilinear superalternating forms for the projective topology of $\operatorname{Vect}(n, m)$; let $\wedge \operatorname{Vect}(n, m)_{0}^{*}$ be the DGA of multilinear superalternating forms for the projective topology of $\operatorname{Vect}(n, m)_{0}^{*}$; see, for instance, [Fuchs 1986]. "Projective" means, in short, that an element of $\operatorname{Vect}(n, m)^{*}$ (respectively, $\left.\operatorname{Vect}(n, m)_{0}^{*}\right)$ is a linear form on $\operatorname{Vect}(n, m)$ that vanishes on all the spaces $\operatorname{Vect}(n, m)^{i}$ (respectively, $\operatorname{Vect}(n, m)_{0}^{i}$ ) but for finitely many $i \in \mathbb{N} \cap\{-1\}$. The spaces $\bigwedge \operatorname{Vect}(n, m)^{*}$ and $\bigwedge \operatorname{Vect}(n, m)_{0}^{*}$ are the exterior products of $\operatorname{Vect}(n, m)^{*}$ and $\operatorname{Vect}(n, m)_{0}^{*}$, respectively.

Denote by $\partial$ be the Chevalley-Eilenberg differential on $\bigwedge \operatorname{Vect}(n, m)^{*}$, and by $\partial_{0}$ the Chevalley-Eilenberg differential on $\bigwedge \operatorname{Vect}(n, m)_{0}^{*}$. The cohomology of the DGA $\left(\bigwedge \operatorname{Vect}(n, m)_{0}^{*}, \partial_{0}\right)$ is called the (Chevalley-Eilenberg) cohomology of $\operatorname{Vect}(n, m)_{0}$, and is denoted by $H^{*}\left(\operatorname{Vect}(n, m)_{0}\right)$. Maybe the name "ChevalleyEilenberg" is not absolutely correct, since we do not consider the complex of all skew-symmetric forms, but there is no risk of confusion here.

Let $\mathscr{E}$ be the ideal (with respect to the product $\wedge$ ) generated by the even elements of $\operatorname{Vect}(n, m)^{*}$, that is, by the continuous linear forms on $\operatorname{Vect}(n, m)$ that identically vanish on $\operatorname{Vect}(n, m)_{0}$. The next lemma will be useful.

Lemma 3.1. The $\operatorname{DGA} \bigwedge \operatorname{Vect}(n, m)^{*} / \mathscr{E}$ and $\bigwedge \operatorname{Vect}(n, m)_{0}^{*}$ are isomorphic.

Remark 3.2. Note that $\operatorname{Vect}(n, m)$ is sometimes denoted by $W(n, m)$. We prefer the notation $\operatorname{Vect}(n, m)$ in order to avoid confusion with the Weil algebra.

3B. Two technical lemmas about the complex $\left(\bigwedge \operatorname{Vect}(n, m)^{*}, \delta\right)$. To prove Proposition 4.2, we need to fix some notation and give the two technical Lemmata 3.5 and 3.6 about the DGA $(\bigwedge \operatorname{Vect}(n, m), \partial)$. 
The family

$$
\left\{\begin{array}{l}
\frac{1}{i_{1} ! \ldots i_{n} !} x_{1}^{i_{1}} \ldots x_{n}^{i_{n}} \theta_{1}^{j_{1}} \ldots \theta_{m}^{j_{m}} \frac{\partial}{\partial x_{i}} \\
\frac{1}{i_{1} ! \ldots i_{n} !} x_{1}^{i_{1}} \ldots x_{n}^{i_{n}} \theta_{1}^{j_{1}} \ldots \theta_{m}^{j_{m}} \frac{\partial}{\partial \theta_{j}}
\end{array}\right.
$$

(with $i_{1}, \ldots, i_{n} \in \mathbb{N}, j_{1}, \ldots, j_{m} \in\{0,1\}, i=1, \ldots, n$, and $j=1, \ldots, m$ ) is a basis of $\operatorname{Vect}(n, m)$. Denote elements of its dual basis by $\left(1 /\left(i_{1} ! \ldots i_{n} !\right) x_{1}^{i_{1}} \ldots x_{n}^{i_{n}} \theta_{1}^{j_{1}} \ldots\right.$ $\left.\theta_{m}^{j_{m}} \partial / \partial x_{i}\right)^{*}$ and $\left(1 /\left(i_{1} ! \ldots i_{n} !\right) x_{1}^{i_{1}} \ldots x_{n}^{i_{n}} \theta_{1}^{j_{1}} \ldots \theta_{m}^{j_{m}} \partial / \partial \theta_{j}\right)^{*}$.

Remark that, if we denote by $\left(x_{1}^{i_{1}} \ldots x_{n}^{i_{n}} \theta_{1}^{j_{1}} \ldots \theta_{m}^{j_{m}} \partial / \partial x_{i}\right)^{*}$ and $\left(x_{1}^{i_{1}} \ldots x_{n}^{i_{n}} \theta_{1}^{j_{1}} \ldots\right.$ $\left.\theta_{m}^{j_{m}} \partial / \partial \theta_{j}\right)^{*}$ the elements of the basis dual to the basis given by $x_{1}^{i_{1}} \ldots x_{n}^{i_{n}} \theta_{1}^{j_{1}} \ldots \theta_{m}^{j_{m}}$ $\partial / \partial x_{i}$ and $x_{1}^{i_{1}} \ldots x_{n}^{i_{n}} \theta_{1}^{j_{1}} \ldots \theta_{m}^{j_{m}} \partial / \partial \theta_{j}$, then we have

$$
\left\{\begin{array}{l}
\left(\frac{1}{i_{1} ! \ldots i_{n} !} x_{1}^{i_{1}} \ldots x_{n}^{i_{n}} \theta_{1}^{j_{1}} \ldots \theta_{m}^{j_{m}} \frac{\partial}{\partial x_{i}}\right)^{*}=i_{1} ! \ldots i_{n} !\left(x_{1}^{i_{1}} \ldots x_{n}^{i_{n}} \theta_{1}^{j_{1}} \ldots \theta_{m}^{j_{m}} \frac{\partial}{\partial x_{i}}\right)^{*} \\
\left(\frac{1}{i_{1} ! \ldots i_{n} !} x_{1}^{i_{1}} \ldots x_{n}^{i_{n}} \theta_{1}^{j_{1}} \ldots \theta_{m}^{j_{m}} \frac{\partial}{\partial \theta_{j}}\right)^{*}=i_{1} ! \ldots i_{n} !\left(x_{1}^{i_{1}} \ldots x_{n}^{i_{n}} \theta_{1}^{j_{1}} \ldots \theta_{m}^{j_{m}} \frac{\partial}{\partial \theta_{j}}\right)^{*}
\end{array}\right.
$$

For convenience, we introduce two notations.

Definition 3.3. For all $i \in\{1, \ldots, n+m\}$, we define $|i|$ to be 0 for $i \in\{1, \ldots, n\}$ and be 1 for $i \in\{n+1, \ldots n+m\}$.

Definition 3.4. We define $h_{i}, i=1, \ldots, n+m$, to be $x_{i}$ if $|i|=0$ and be $\theta_{i-n}$ if $|i|=1$.

For example, we denote by $h_{i_{1}} \ldots h_{i_{s}} h_{n+j_{1}} \ldots h_{n+j_{t}} \partial / \partial h_{1} \in \operatorname{Vect}(n, m)$ the element that we used to denote by $x_{i_{1}} \ldots x_{i_{s}} \theta_{j_{1}} \ldots \theta_{j_{t}} \partial / \partial x_{1}$, where $i_{1}, \ldots, i_{s} \in\{1, \ldots$, $n\}$ and $j_{1}, \ldots, j_{t} \in\{1, \ldots, m\}$.

Define the weight of $\left(h_{i_{1}} \ldots h_{i_{k}} \partial / \partial h_{i}\right)^{*}$ to be $k-1$ for any $i_{1}, \ldots, i_{k} \in\{1$, $\ldots, n+m\}$. Define $\Lambda_{k}$ to be the subalgebra of $\bigwedge \operatorname{Vect}(n, m)_{0}^{*}$ generated (with respect to $\wedge$ ) by elements of weights $-1,0,1, \ldots, k$. One has $\partial \Lambda_{k-1} \subset \Lambda_{k}$.

We now compute the Chevalley-Eilenberg differential in the basis dual to the basis in (3-2). For this purpose, we define $c_{j}^{i_{1}, \ldots, i_{k}}$ for any $i_{1}, \ldots, i_{k}, j \in\{1, \ldots$, $n+m\}$ by

$$
c_{j}^{i_{1}, \ldots, i_{k}}=\prod_{i=1}^{n} K\left(i,\left[i_{1}, \ldots, i_{k}\right]\right) !\left(h_{i_{1}} \ldots h_{i_{k}} \frac{\partial}{\partial h_{j}}\right)^{*},
$$

where $K\left(i,\left[i_{1}, \ldots, i_{k}\right]\right)$ is the number of integers equal to $i$ in the list $\left[i_{1}, \ldots, i_{k}\right]$. If $|j|=1$ and $j$ appears more than twice in the list $\left[i_{1}, \ldots, i_{k}\right]$, then, of course,

$$
c_{j}^{i_{1}, \ldots, i_{k}}=0 .
$$


Otherwise, we have

$$
c_{j}^{i_{1}, \ldots, i_{k}}=\varepsilon\left(\frac{1}{k_{1} ! \ldots k_{n} !} x_{1}^{k_{1}} \ldots x_{n}^{k_{n}} \theta_{1}^{j_{1}} \ldots \theta_{m}^{j_{m}} \frac{\partial}{\partial h_{j}}\right)^{*}
$$

for some $\varepsilon \in\{-1,1\}$, where $k_{1}=K\left(1,\left[i_{1}, \ldots, i_{k}\right]\right), \ldots, k_{n}=K\left(n,\left[i_{1}, \ldots, i_{k}\right]\right)$ and where, for any $i \in\{1, \ldots, m\}, j_{i}=1$ if and only if $n+i$ appears in the list $\left[i_{1}, \ldots, i_{k}\right]$.

For any $i_{1}, \ldots, i_{k}, j \in\{1, \ldots, n+m\}$, we define $P_{j}^{i_{1}, \ldots, i_{k}} \in \Lambda_{k-1}$ by:

$$
\partial c_{j}^{i_{1}, \ldots, i_{k}}=P_{j}^{i_{1}, \ldots, i_{k}}+\sum_{l=1}^{n+m}(-1)^{|l|}\left(\frac{\partial}{\partial h_{l}}\right)^{*} \wedge c_{j}^{l, i_{1}, \ldots, i_{k}} .
$$

Lemma 3.5. For any $i_{1}, \ldots, i_{k}, j \in\{1, \ldots, n+m\}, P_{j}^{i_{1}, \ldots, i_{k}}$ is an element of $\Lambda_{k-1}$. Proof. Denote by $\Sigma_{l, k}$ the set of shuffles of the sets $\{1, \ldots, l\}$ and $\{l+1, \ldots, k\}$. We have

$$
\partial c_{j}^{i_{1}, \ldots, i_{k}}=\sum_{l=1}^{n+m} \sum_{\sigma \in \Sigma_{l, k}} D\left(i_{1}, \ldots, i_{k}, l, j, \sigma\right) c_{l}^{i_{\sigma(l+1)}, \ldots, i_{\sigma(k)}} \wedge c_{j}^{l, i_{\sigma(1)}, \ldots, i_{\sigma(l)}},
$$

for some $D\left(i_{1}, \ldots, i_{k}, l, j, \sigma\right) \in \mathbb{R}$.

From the relations

$$
\begin{aligned}
& {\left[\frac{\partial}{\partial x_{i}}, \frac{1}{k_{1} ! \ldots\left(k_{i}+1\right) ! \ldots k_{n} !} x_{1}^{k_{1}} \ldots x_{i}^{k_{i}+1} \ldots x_{n}^{k_{n}} \theta_{1}^{j_{1}} \ldots \theta_{m}^{j_{m}} \frac{\partial}{\partial h_{j}}\right]} \\
& =\frac{1}{k_{1} ! \ldots k_{i} ! \ldots k_{n} !} x_{1}^{k_{1}} \ldots x_{i}^{k_{i}} \ldots x_{n}^{k_{n}} \theta_{1}^{j_{1}} \ldots \theta_{m}^{j_{m}} \frac{\partial}{\partial h_{j}}
\end{aligned}
$$

$$
\begin{aligned}
{\left[\frac{\partial}{\partial \theta_{l}}, \frac{\theta_{l}}{k_{1} ! \ldots k_{n} !} x_{1}^{k_{1}} \ldots\right.} & \left.x_{n}^{k_{n}} \theta_{1}^{j_{1}} \ldots \theta_{l-1}^{j_{l-1}} \theta_{l+1}^{j_{l+1}} \ldots \theta_{m}^{j_{m}} \frac{\partial}{\partial h_{j}}\right] \\
& =\frac{1}{k_{1} ! \ldots k_{n} !} x_{1}^{k_{1}} \ldots x_{n}^{k_{n}} \theta_{1}^{j_{1}} \ldots \theta_{l-1}^{j_{l-1}} \theta_{l+1}^{j_{l+1}} \ldots \theta_{m}^{j_{m}} \frac{\partial}{\partial h_{j}}
\end{aligned}
$$

we obtain, using (3-1), (3-4), and the definition of $\partial$, that

$$
\partial c_{j}^{i_{1}, \ldots, i_{k}}-\sum_{l=1}^{n+m}(-1)^{|l|}\left(\frac{\partial}{\partial h_{l}}\right)^{*} \wedge c_{j}^{l, i_{1}, \ldots, i_{k}}
$$

is a linear combinations of products of elements of weight $0, \ldots, k-1$ that do not involve any terms of weight -1 or $k$. In other words, it is an element of $\Lambda_{k-1}$.

Lemma 3.6. For any $i_{1}, \ldots, i_{k}, l \in\{1, \ldots, n+m\}$, define 2 -forms $Q_{j}^{l, i_{1}, \ldots, i_{k}} \in \Lambda_{k}$ by

$$
Q_{j}^{l, i_{1}, \ldots, i_{k}}=P_{j}^{l, i_{1}, \ldots, i_{k}}-\sum_{l^{\prime}=1}^{n+m}(-1)^{|l|+\left|l^{\prime}\right|}\left(h_{l} \frac{\partial}{\partial h_{l^{\prime}}}\right)^{*} \wedge c_{j}^{l^{\prime}, i_{1}, \ldots, i_{k}}
$$


The following identity holds:

$$
\partial P_{j}^{i_{1}, \ldots, i_{k}}=\sum_{l=1}^{n+m}\left(\frac{\partial}{\partial h_{l}}\right)^{*} \wedge Q_{j}^{l, i_{1}, \ldots, i_{k}} .
$$

Proof. The identity

$$
\partial c_{i}=\sum_{j=1}^{n+m}(-1)^{|j|} c_{j} \wedge c_{i}^{j}
$$

holds for any $i \in\{1, \ldots, n+m\}$. Applying $\partial$ to (3-5) and using (3-11), we obtain

$$
\begin{aligned}
& \partial P_{j}^{i_{1}, \ldots, i_{k}}+\partial \sum_{l=1}^{n+m}(-1)^{|l|}\left(\frac{\partial}{\partial h_{l}}\right)^{*} \wedge c_{j}^{l, i_{1}, \ldots, i_{k}}=0, \\
& \partial P_{j}^{i_{1}, \ldots, i_{k}}+\sum_{l=1}^{n+m} \sum_{l^{\prime}=1}^{n+m}(-1)^{\left|l^{\prime}\right|+|l|}\left(\frac{\partial}{\partial h_{l^{\prime}}}\right)^{*} \wedge\left(h_{l^{\prime}} \frac{\partial}{\partial h_{l}}\right)^{*} \wedge c_{j}^{l, i_{1}, \ldots, i_{k}}, \\
&-\sum_{l=1}^{n+m}\left(\frac{\partial}{\partial h_{l}}\right)^{*} \wedge \partial c_{j}^{l, i_{1}, \ldots, i_{k}}=0,
\end{aligned}
$$$$
\partial P_{j}^{i_{1}, \ldots, i_{k}}+\sum_{l=1}^{n+m} \sum_{l^{\prime}=1}^{n+m}(-1)^{\left|l^{\prime}\right|+|l|}\left(\frac{\partial}{\partial h_{l}}\right)^{*} \wedge\left(h_{l} \frac{\partial}{\partial h_{l^{\prime}}}\right)^{*} \wedge c_{j}^{l^{\prime}, i_{1}, \ldots, i_{k}}-\sum_{l=1}^{n+m}\left(\frac{\partial}{\partial h_{l}}\right)^{*} \wedge P_{j}^{l, i_{1}, \ldots, i_{k}}
$$$$
-\sum_{l^{\prime}=1}^{n+m} \sum_{l=1}^{n+m}(-1)^{\left|l^{\prime}\right|}\left(\frac{\partial}{\partial h_{l}}\right)^{*} \wedge\left(\frac{\partial}{\partial h_{l^{\prime}}}\right)^{*} \wedge c_{j}^{l^{\prime}, l, i_{1}, \ldots, i_{k}}=0 .
$$

Now, for any $l^{\prime}, l \in\{1, \ldots, n+m\}$, we have

$$
\left\{\begin{array}{l}
\left(\frac{\partial}{\partial h_{l}}\right)^{*} \wedge\left(\frac{\partial}{\partial h_{l^{\prime}}}\right)^{*}=(-1)^{(|l|+1)\left(\left|l^{\prime}\right|+1\right)}\left(\frac{\partial}{\partial h_{l^{\prime}}}\right)^{*} \wedge\left(\frac{\partial}{\partial h_{l}}\right)^{*} \\
c_{j}^{l^{\prime}, l, i_{1}, \ldots, i_{k}}=(-1)^{\left|l^{\prime}\right||l|} c_{j}^{l, l^{\prime}, i_{1}, \ldots, i_{k}}
\end{array}\right.
$$

Therefore, we have

$$
(-1)^{\left|l^{\prime}\right|}\left(\frac{\partial}{\partial h_{l}}\right)^{*} \wedge\left(\frac{\partial}{\partial h_{l^{\prime}}}\right)^{*} \wedge c_{j}^{l^{\prime}, l, i_{1}, \ldots, i_{k}}=-(-1)^{|l|}\left(\frac{\partial}{\partial h_{l^{\prime}}}\right)^{*} \wedge\left(\frac{\partial}{\partial h_{l}}\right)^{*} \wedge c_{j}^{l, l^{\prime}, i_{1}, \ldots, i_{k}}
$$

and

$$
\sum_{l^{\prime}=1}^{n+m} \sum_{l=1}^{n+m}(-1)^{\left|l^{\prime}\right|}\left(\frac{\partial}{\partial h_{l}}\right)^{*} \wedge\left(\frac{\partial}{\partial h_{l^{\prime}}}\right)^{*} \wedge c_{j}^{l^{\prime}, l, i_{1}, \ldots, i_{k}}=0 .
$$

The lemma follows immediately.

3C. Generators of the cohomology of $\operatorname{Vect}(\boldsymbol{n}, \boldsymbol{m})_{0}$. We introduce a DGA whose cohomology is $H^{*}\left(\operatorname{Vect}(n, m)_{0}\right)$ but that is easier to work with than $\operatorname{Vect}(n, m)_{0}$ itself.

Let $g l_{n}$ (respectively, $g l_{m}$ ) be the Lie algebra of linear endomorphism of $\mathbb{R}^{n}$ (respectively, $\left.\mathbb{R}^{m}\right)$. Let $\left(a_{i, j}\right)_{i, j \in\{1, \ldots, n\}}$ (respectively, $\left.\left(d_{k, l}\right)_{k, l \in\{1, \ldots, m\}}\right)$ be the canonical basis of $g l_{n}$ (respectively, $g l_{m}$ ). 
The elements $\operatorname{Vect}(n, m)_{0}^{0}$ of weight 0 in $\operatorname{Vect}(n, m)_{0}$ form a Lie subalgebra of $\operatorname{Vect}(n, m)_{0}$, isomorphic to $g l_{n} \oplus g l_{m}$ through the isomorphism $\varphi$ with

$$
\varphi\left(x_{i} \frac{\partial}{\partial x_{j}}\right)=a_{i, j} \quad \text { and } \quad \varphi\left(\theta_{k} \frac{\partial}{\partial \theta_{l}}\right)=d_{k, l} .
$$

Let $p_{0}$ be the projection from $\operatorname{Vect}(n, m)_{0}$ to $\operatorname{Vect}(n, m)_{0}^{0}$, with kernel

$$
\operatorname{Ker} p_{0}=\operatorname{Vect}(n, m)_{0}^{-1} \oplus \bigoplus_{i=1}^{\infty} \operatorname{Vect}(n, m)_{0}^{i} \text {. }
$$

Let $\alpha:\left(g l_{n} \oplus g l_{m}\right)^{*} \rightarrow \operatorname{Vect}(n, m)_{0}^{*}$ be the linear map $\alpha=p_{0}^{*} \circ \varphi^{*}$, where $p_{0}^{*}$ and $\varphi^{*}$ are the dual maps of $p_{0}$ and $\varphi$.

The map $\alpha$ defines a $g l_{n} \oplus g l_{m}$-connection (see [Guillemin and Sternberg 1999, Chapter 3]) of the DGA $\bigwedge \operatorname{Vect}(n, m)_{0}^{*}$ that induces a DGA homomorphism (denoted again by $\alpha)$ from the Weil algebra $W\left(g l_{n} \oplus g l_{m}\right)=S\left(\left(g l_{n} \oplus g l_{m}\right)^{*}\right) \otimes \wedge\left(g l_{n} \oplus g l_{m}\right)^{*}$ to $\bigwedge \operatorname{Vect}(n, m)_{0}^{*}$.

Let $\mathscr{K}$ be the kernel of $\alpha$. The cohomology of the DGA $W\left(g l_{n} \oplus g l_{m}\right) / \mathscr{K}$ is denoted by $H^{*}\left(W\left(g l_{n} \oplus g l_{m}\right) / \mathscr{K}\right)$. Write $\tilde{\alpha}$ for the DGA homomorphism from $W\left(g l_{n} \oplus g l_{m}\right) / \mathscr{F}$ to $\bigwedge \operatorname{Vect}(n, m)_{0}^{*}$ induced by $\alpha$. Let $\hat{\alpha}: H^{*}\left(W\left(g l_{n} \oplus g l_{m}\right) / \mathscr{K}\right) \rightarrow$ $H^{*}\left(\operatorname{Vect}(n, m)_{0}\right)$ be the map induced by $\tilde{\alpha}$ in cohomology.

Theorem 3.7. The map $\hat{\alpha}$ is an isomorphism between $H^{*}\left(W\left(g l_{n} \oplus g l_{m}\right) / \mathscr{K}\right)$ and $H^{*}\left(\operatorname{Vect}(n, m)_{0}\right)$.

The proof is provided in Appendix A.

\section{Secondary characteristic classes of superfoliations}

We construct, for any superfoliation $\mathscr{F}$ on $\mathcal{M}$ of codimension $n+\varepsilon m$ with trivialized normal bundle, a map from $H^{*}\left(\operatorname{Vect}(n, m)_{0}\right)$ to the de Rham cohomology of the base manifold $H^{*}(M)$.

We use the conventions introduced in Defintions 3.3 and 3.4. Throughout this section, we often say "homomorphism" for "DGA homomorphism".

\section{A. DGA homomorphism defining a superfoliation.}

Definition 4.1. We say that a DGA homomorphism

$$
\omega:\left(\bigwedge \operatorname{Vect}(n, m)^{*}, \partial\right) \rightarrow\left(\Omega^{*}(\mathcal{M}), d_{M}\right)
$$

defines the superfoliation $\mathscr{F}$ of codimension $n+\varepsilon m$ with trivialized normal bundle if the 1 -forms $\omega\left(\left(\partial / \partial h_{i}\right)^{*}\right), i=1, \ldots, n+m$, define the superfoliation $\mathscr{F}$.

Consider a superfoliation $\mathscr{F}_{F}$ with trivialized normal bundle on $M$, defined by 1 -forms $b_{1}, \ldots, b_{n+m}$, where $b_{1}, \ldots, b_{n}$ are odd 1 -forms and $b_{n+1}, \ldots, b_{n+m}$ are 
even 1-forms. Take 1-forms $\left(b_{i}^{j}\right)_{i, j \in\{1, \ldots, n+m\}}$ such that

$$
d_{M} b_{i}=\sum_{j=1}^{n} b_{j} \wedge b_{i}^{j}-\sum_{j=n+1}^{n+m} b_{j} \wedge b_{i}^{j} .
$$

According to (2-6), such 1-forms $b_{i}^{j}$ always exist. The choice of signs in (4-1) is explained by:

Proposition 4.2. For any superfoliation $\mathscr{F}$ with trivialized normal bundle on $\mathcal{M}$, there exists a DGA homomorphism $\omega$ from $\left(\bigwedge \operatorname{Vect}(n, m)^{*}, \partial\right)$ to $\left(\Omega(\mathcal{M}), d_{\mu}\right)$ that defines the superfoliation $\mathscr{F}$. Moreover, we can assume that

$$
\begin{aligned}
\omega\left(\left(\partial / \partial h_{i}\right)^{*}\right) & =b_{i}, & i=1, \ldots, n+m \\
\omega\left(\left(h_{i} \partial / \partial h_{j}\right)^{*}\right) & =b_{j}^{i}, & i, j=1, \ldots, n+m .
\end{aligned}
$$

First, we need the following lemma, proved in Appendix B.

Lemma 4.3. Let $d_{1}, \ldots, d_{n}$ be odd 1 -forms and $d_{n+1}, \ldots, d_{n+m}$ even 1-forms, forming a free family. Let $d^{i}, i=1, \ldots, n+m$, be 2 -forms on $M$ such that

$$
\sum_{i=1}^{n+m} d_{i} \wedge d^{i}=0
$$

There exist homogeneous 1 -forms $d^{i, l}$ of parity $|i|+|l|+1$ (modulo 2) such that

$$
d^{i, l}=-(-1)^{(|i|+1)(|l|+1)} d^{l, i} \quad \text { and } \quad \sum_{i=1}^{n+m} d_{i} \wedge d^{i, l}=d^{l} .
$$

Proof of Proposition 4.2. Define $\varepsilon\left(i_{1}, \ldots, i_{k}, \sigma\right) \in\{-1,1\}$, for any permutation $\sigma$ of $\{1, \ldots, k\}$, by

$$
h_{i_{\sigma(1)}} \ldots h_{i_{\sigma(k)}}=\varepsilon\left(i_{1}, \ldots, i_{k}, \sigma\right) h_{i_{1}} \ldots h_{i_{k}} .
$$

We will construct by induction a homomorphism $\omega: \Lambda_{k} \rightarrow \Omega(\mathcal{M})$ such that, for any $\alpha \in \Lambda_{k-1}$,

$$
d_{M} \omega(\alpha)=\omega(\partial(\alpha)) .
$$

Equations (4-2) and (4-3) define such a map $\omega$ for $k=0$. Equations (3-11) and (4-1) imply that the condition (4-4) holds for $k=0$. We have therefore constructed $\omega$ for $k=0$.

We now assume that $\omega$ can be constructed for some $k \in \mathbb{N}$ and construct it for $k+1$. Applying $\omega$ to (3-5), we obtain

$$
d_{M} \omega\left(c_{j}^{i_{1}, \ldots, i_{k}}\right)=\omega\left(P_{j}^{i_{1}, \ldots, i_{k}}\right)+\sum_{l=1}^{n+m}(-1)^{|l|} \omega\left(\left(\partial / \partial h_{l}\right)^{*}\right) \wedge \omega\left(c_{j}^{l, i_{1}, \ldots, i_{k}}\right) .
$$


Denote $\omega\left(c_{j}^{i_{1}, \ldots, i_{n}}\right)$ by $b_{j}^{i_{1}, \ldots, i_{n}}$. In particular, $b_{l}=\omega\left(\left(\partial / \partial x_{l}\right)^{*}\right)$. By applying $d_{\mu}$ to both sides of the previous expression and using Equation (4-2), we obtain

$$
d_{M} \omega\left(P_{j}^{i_{1}, \ldots, i_{k}}\right)+d_{M}\left(\sum_{l=1}^{n+m}(-1)^{|l|} b_{l} \wedge b_{j}^{l, i_{1}, \ldots, i_{k}}\right)=0 .
$$

By (4-1), we have

$$
d_{M} \omega\left(P_{j}^{i_{1}, \ldots, i_{k}}\right)+\sum_{i=1}^{n+m} b_{i} \wedge\left(\sum_{l=1}^{n+m}(-1)^{|i|+|l|} b_{l}^{i} \wedge b_{j}^{l, i_{1}, \ldots, i_{k}}-d_{M} b_{j}^{i, i_{1}, \ldots, i_{k}}\right)=0 .
$$

Since $P_{j}^{i_{1}, \ldots, i_{k}} \in \Lambda_{k-1}$, we have $\omega\left(\partial P_{j}^{i_{1}, \ldots, i_{k}}\right)=d_{\mathcal{M}} \omega\left(P_{j}^{i_{1}, \ldots, i_{k}}\right)$. Equation (3-10) implies that

$$
\sum_{i=1}^{n+m} b_{i} \wedge\left(\omega\left(Q_{j}^{i, i_{1}, \ldots, i_{k}}\right)+\sum_{l=1}^{n+m}(-1)^{|l|+|i|} b_{l}^{i} \wedge b_{j}^{l, i_{1}, \ldots, i_{k}}-d_{M} b_{j}^{i, i_{1}, \ldots, i_{k}}\right)=0 .
$$

From (3-9) it follows that

$$
\sum_{i=1}^{n+m} b_{i} \wedge\left(\omega\left(P_{j}^{i, i_{1}, \ldots, i_{k}}\right)-d_{M} c_{j}^{i, i_{1}, \ldots, i_{k}}\right)=0 .
$$

By Lemma 4.3, there exist 1 -forms $b_{l}^{i, l, i_{1}, \ldots, i_{k}}$ satisfying

$$
\left\{\begin{array}{l}
d_{M} b_{j}^{i, i_{1}, \ldots, i_{k}}-\omega\left(P_{j}^{i, i_{1}, \ldots, i_{k}}\right)=\sum_{l=1}^{n+m}(-1)^{|l|} b_{l} \wedge b_{j}^{l, i, i_{1}, \ldots, i_{k}} \\
(-1)^{|i|} b_{j}^{i, l, i_{1}, \ldots, i_{k}}=-(-1)^{(|l|+1)(|i|+1)}(-1)^{|l|} b_{j}^{l, i, i_{1}, \ldots, i_{k}} .
\end{array}\right.
$$

This last equation can be rewritten

$$
b_{j}^{i, l, i_{1}, \ldots, i_{k}}=(-1)^{|i||l|} b_{j}^{l, i, i_{1}, \ldots, i_{k}} .
$$

Equation (4-5) and the relations

$$
\left\{\begin{array}{l}
b_{j}^{i_{\sigma(1)}, \ldots, i_{\sigma(k)}, i_{\sigma(k+1)}}=\varepsilon\left(i_{1}, \ldots, i_{k+1}, \sigma\right) b_{j}^{i_{1}, \ldots, i_{k}, i_{k+1}} \\
P_{j}^{i_{\sigma(1)}, \ldots, i_{\sigma(k)}, i_{\sigma(k+1)}}=\varepsilon\left(i_{1}, \ldots, i_{k+1}, \sigma\right) P_{j}^{i_{1}, \ldots, i_{k}, i_{k+1}} .
\end{array}\right.
$$

imply that, for any permutation $\sigma$ of $\{1, \ldots, k+1\}$,

$$
b_{j}^{i, i_{\sigma(1)}, \ldots, i_{\sigma(k+1)}}=\varepsilon\left(i_{1}, \ldots, i_{k+1}, \sigma\right) b_{j}^{i, i_{1}, \ldots, i_{k+1}} .
$$

Equations (4-6) and (4-7) imply that, for any permutation $\sigma$ of $\{1, \ldots, k+2\}$ and any $i_{1}, \ldots, i_{k+1}, i_{k+2} \in\{1, \ldots, n+m\}$, we have

$$
b_{j}^{i_{\sigma(1)}, \ldots, i_{\sigma(k+1)}, i_{\sigma(k+2)}}=\varepsilon\left(i_{1}, \ldots, i_{k}, i_{k+2}, \sigma\right) b_{j}^{i_{1}, \ldots, i_{k+1}, i_{k+2}} .
$$


We can then define $\omega$ on elements of weight $k+1$ by $\omega\left(c_{j}^{i_{1}, \ldots, i_{k+2}}\right)=b_{j}^{i_{1}, \ldots, i_{k+2}}$. Equation (4-8) implies that

$$
\omega\left(c_{j}^{\left.i_{\sigma(1)}, \ldots, i_{\sigma(k+2)}\right)}\right)=\varepsilon\left(i_{1}, \ldots, i_{k+2}, \sigma\right) \omega\left(c_{j}^{i_{1}, \ldots, i_{k+2}}\right),
$$

and hence $\omega$ is well defined. The map $\omega$ can be uniquely extended to a DGA homomorphism from $\Lambda_{k+1}$ to $\Omega(M)$. According to (3-5) and (4-5), this homomorphism satisfies $d_{M} \omega(\alpha)=\omega(\partial(\alpha))$ for any $\alpha \in \Lambda_{k}$. This completes the proof.

4B. Construction of secondary characteristic classes. Assume that

$$
\omega: \bigwedge \operatorname{Vect}(n, m)_{0}^{*} \rightarrow \Omega(\mathcal{M})
$$

is a DGA homomorphism defining a superfoliation $\mathscr{F}$ of codimension $n+\varepsilon m$ with trivialized normal bundle. Set $\beta_{\omega}=p \circ \omega$, where $p$ is the canonical projection from $\Omega^{*}(M)$ to $\Omega^{*}(M)$. By Lemma 2.1, the kernel of $p$ contains all odd $2 k$-forms and even $(2 k+1)$-forms. As a consequence, $\omega(\mathscr{E})$ is contained in the kernel of $\beta_{\omega}$. The homomorphism $\beta_{\omega}$ induces a homomorphism from $\wedge \operatorname{Vect}(n, m)^{*} / \mathscr{E}$ to $\Omega^{*}(M)$. By Lemma 3.1, $\bigwedge \operatorname{Vect}(n, m)^{*} / \mathscr{E} \simeq \bigwedge \operatorname{Vect}(n, m)_{0}^{*}$, and therefore $\beta_{\omega}$ induces a homomorphism $\beta_{\omega}^{\prime}$ from $\wedge \operatorname{Vect}(n, m)_{0}^{*}$ to $\Omega^{*}(M)$.

Definition 4.4. Let $\varphi_{\mathcal{M}, \mathscr{F}}$ be the homomorphism from $H^{*}\left(\operatorname{Vect}(n, m)_{0}\right)$ to $H^{*}(M)$ induced in cohomology by the DGA homomorphism

$$
\beta_{\omega}^{\prime}: \bigwedge \operatorname{Vect}(n, m)_{0}^{*} \rightarrow \Omega^{*}(M)
$$

This definition is justified by:

Proposition 4.5. The homomorphism $\varphi_{\mathcal{M}, \mathscr{F}}: H^{*}\left(\operatorname{Vect}(n, m)_{0}\right) \rightarrow H^{*}(M)$ is independent of the choice of homomorphism $\omega: \bigwedge \operatorname{Vect}(n, m)^{*} \rightarrow \Omega(M)$ defining the superfoliation $\mathscr{F}$.

First, we need a lemma. Let $\omega^{1}$ and $\omega^{2}$ be two homomorphisms defining the superfoliation $\mathscr{F}$, and let $\varphi_{\mathcal{M}, \mathscr{F}}^{1}, \varphi_{\mathcal{M}, \mathscr{F}}^{2}$ be the homomorphisms $\wedge \operatorname{Vect}(n, m)^{*} \rightarrow$ $\Omega(\mathcal{M})$ constructed from $\omega_{1}$ and $\omega_{2}$ as in Definition 4.4.

Lemma 4.6. If $p \circ \omega^{1}\left(\left(x_{i} \partial / \partial x_{j}\right)^{*}\right)=p \circ \omega^{2}\left(\left(x_{i} \partial / \partial x_{j}\right)^{*}\right)$ and $p \circ \omega^{1}\left(\left(\theta_{k} \partial / \partial \theta_{l}\right)^{*}\right)=$ $p \circ \omega^{2}\left(\left(\theta_{k} \partial / \partial \theta_{l}\right)^{*}\right) \in \Omega(M)$ for all $i, j \in\{1, \ldots, n\}$ and $k, l \in\{1, \ldots, m\}$, then

$$
\varphi_{\mathcal{M}, \mathscr{F}}^{1}=\varphi_{\mathcal{M}, \mathscr{F}}^{2} .
$$

Proof. A DGA homomorphism $\beta$ from the Weil algebra $W(\mathfrak{g})=S\left(\mathfrak{g}^{*}\right) \otimes \wedge \mathfrak{g}^{*}$ of a Lie algebra $\mathfrak{g}$ to a given DGA $\left(A, d_{A}\right)$ depends only on the restriction of $\beta$ to $1 \otimes \bigwedge^{1} \mathfrak{g}^{*}$, see [Guillemin and Sternberg 1999]. In particular, the homomorphisms $\beta_{\omega^{a}} \circ \alpha: W\left(g l_{n} \oplus g l_{m}\right) \rightarrow \Omega(M)$ for $a=1,2$ depend only on the 
1-forms $p \circ \omega\left(\left(x_{i} \partial / \partial x_{j}\right)^{*}\right)$ and $p \circ \omega\left(\left(\theta_{k} \partial / \partial \theta_{l}\right)^{*}\right)$, where $i, j \in\{1, \ldots, n\}$ and $k, l \in\{1, \ldots, m\}$. Therefore,

$$
\beta_{\omega^{1}} \circ \alpha=\beta_{\omega^{2}} \circ \alpha \quad \text { and } \quad \beta_{\omega^{1}}^{\prime} \circ \tilde{\alpha}=\beta_{\omega^{2}}^{\prime} \circ \tilde{\alpha} .
$$

For $a=1,2$, the diagram

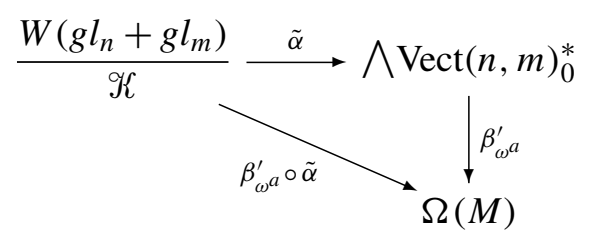

is commutative. Moreover, according to Theorem 3.7, $\tilde{\alpha}$ induces an isomorphism in cohomology. This completes the proof.

Proof of Proposition 4.5. Set $a_{i}=\omega^{1}\left(\left(\partial / \partial h_{i}\right)^{*}\right)$ and $b_{i}=\omega^{2}\left(\left(\partial / \partial h_{i}\right)^{*}\right)$. Set also $a_{i}^{j}=\omega^{1}\left(\left(h_{j} \partial / \partial h_{i}\right)^{*}\right)$ and $b_{i}^{j}=\omega^{2}\left(\left(h_{j} \partial / \partial h_{i}\right)^{*}\right)$.

For any superfunction $f$ on the supermanifold $\mu \times \mathbb{R}$, denote by $\left.f\right|_{t}$ its restriction to $M \times\{t\}$. Denote by $i_{t}$ the maps from $M$ to $M \times \mathbb{R}$ induced by $\left.f \rightarrow f\right|_{t}$. Let

$$
\text { pr }: M \times \mathbb{R} \rightarrow M
$$

be the natural projection.

On the supermanifold $M \times \mathbb{R}$, consider the superfoliation $\mathscr{F}^{\prime}$ that is the pullback of $\mathscr{F}$ by the projection pr. There exist 1 -forms $c_{1}, \ldots, c_{n+m}$ defining the superfoliation $\mathscr{F}^{\prime}$ and 1 -forms $\left(c_{i}^{k}\right)_{i, k \in\{1, \ldots, n+m\}}$ satisfying the identity

$$
d_{M} c_{i}=\sum_{k=1}^{n+m}(-1)^{|k|} c_{k} \wedge c_{i}^{k}
$$

and the following two properties:

- The 1 -forms $c_{i}, i \in\{1, \ldots, n+m\}$ (respectively, $c_{i}^{k}, i, k \in\{1, \ldots, n+m\}$ ) restricted to $\mathcal{M} \times(-\infty, 1 / 4]$ are equal to $\mathrm{pr}^{*} a_{i}, i=1, \ldots, n+m$ (respectively, $\left.\operatorname{pr}^{*} a_{k}^{i}, i, k \in\{1, \ldots, n+m\}\right)$.

- The 1 -forms $c_{i}, i \in\{1, \ldots, n+m\}$ (respectively, $c_{i}^{k}, i, k \in\{1, \ldots, n+m\}$ ) restricted to $M \times(3 / 4, \infty)$ are equal to $\operatorname{pr}^{*} b_{i}, i=1, \ldots, n+m$ (respectively, $\left.\operatorname{pr}^{*} b_{k}^{i}, i, k \in\{1, \ldots, n+m\}\right)$.

By Proposition 4.2, there exists a homomorphism $\omega_{3}: \bigwedge \operatorname{Vect}(n, m)^{*} \rightarrow \Omega(M \times \mathbb{R})$ defining $\mathscr{F}^{\prime}$, such that $\omega_{3}\left(\left(h_{i} \partial / \partial h_{j}\right)^{*}\right)=c_{j}^{i}$ and $\omega_{3}\left(\left(\partial / \partial h_{i}\right)^{*}\right)=c_{i}$. Then, the 
following diagram is commutative:

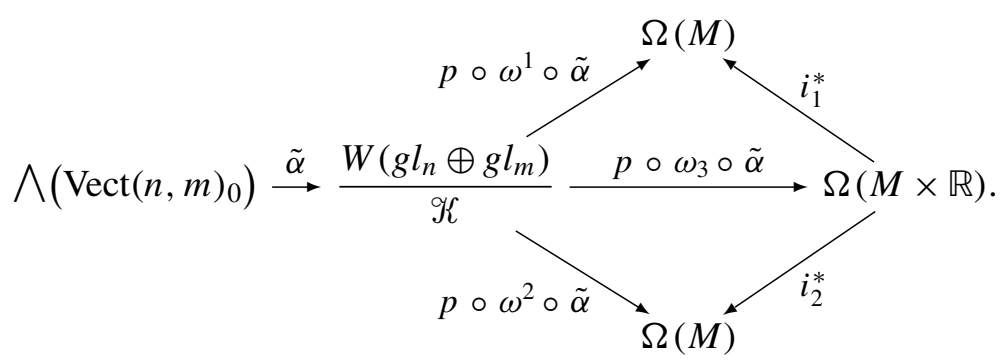

Therefore, we also have the commutative diagram

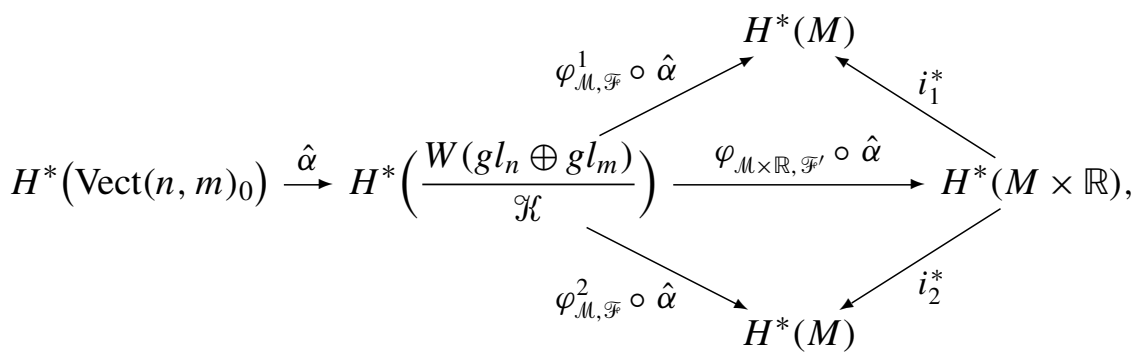

where $\varphi_{\mathcal{M} \times \mathbb{R}, \mathscr{F}^{\prime}}: H^{*}(\operatorname{Vect}(n, m))$ is constructed as in Definition 4.4, with the help of the DGA homomorphism $\omega_{3}$ defining $\mathscr{F}^{\prime}$.

Since by Theorem $3.7 \hat{\alpha}$ is a bijection, and $i_{1}^{*}: H^{*}(\mathcal{M} \times \mathbb{R}) \rightarrow H^{*}(\mathcal{M})$ and $i_{2}^{*}: H^{*}(M \times \mathbb{R}) \rightarrow H^{*}(\mathcal{M})$ are isomorphisms with $i_{1}^{*} \circ\left(i_{2}^{*}\right)^{-1}=\mathrm{Id}$, we have

$$
\varphi_{\mathcal{M}, \mathscr{F}}^{1}=\varphi_{\mathcal{M}, \mathscr{F}}^{2} .
$$

We now study how $\varphi_{\mathcal{M}, \mathscr{F}}$ behaves with respect to morphisms of superfoliations.

Proposition 4.7. Let $\mathscr{F}$ (respectively, $\mathscr{G}$ ) be a superfoliation with trivialized normal bundle on $\mathcal{M}$ (respectively, $\mathcal{N}$ ). Let $\Phi: \mathcal{N} \rightarrow \mathcal{M}$ be a morphism of foliated supermanifolds, and $\varphi: N \rightarrow M$ the map induced by $\Phi$, from the base manifold $N$ of $\mathcal{N}$ to the base manifold $M$ of $\mathcal{N}$. Let $\varphi^{*}: H^{*}(M) \rightarrow H^{*}(N)$ be the pull-pack by $\varphi$. Under these hypotheses, we have

$$
\varphi_{\mathcal{N}, \mathscr{G}}=\varphi^{*} \circ \varphi_{\mathcal{M}, \mathscr{F}} .
$$

In other words, for any supermanifold $\mathcal{N}$ and any submersion $\Phi: \mathcal{N} \rightarrow \mathcal{M}$, we have $\varphi_{\mathcal{N}, \Phi^{*} \mathscr{F}_{F}}=\varphi^{*} \circ \varphi_{\mathcal{M}, \mathscr{F}}$, where $\Phi^{*} \mathscr{F}_{F}$ is the pull-back via $\Phi$ of the superfoliation $\mathscr{F}$.

Proof. If $\omega: \bigwedge \operatorname{Vect}(n, m)^{*} \rightarrow \Omega(\mathcal{M})$ is a DGA homomorphism defining $\mathscr{F}$, then $\Phi^{*} \circ \omega: \bigwedge \operatorname{Vect}(n, m)^{*} \rightarrow \Omega(\mathcal{N})$ is a homomorphism of DGAs defining $\mathscr{G}$. Let $p_{N}: \Omega(\mathcal{N}) \rightarrow \Omega(N)$ and $p_{M}: \Omega(\mathcal{N}) \rightarrow \Omega(N)$ be the canonical projections of the supermanifolds $\mathcal{N}$ and $\mathcal{M}$ to their base manifolds, as defined in Lemma 2.1. We 
have $p_{N} \circ \Phi^{*}=\varphi^{*} \circ p_{M}$, which implies that

$$
p_{N} \circ \Phi^{*} \circ \omega=\varphi^{*} \circ p_{M} \circ \omega .
$$

Hence, $\beta_{\Phi^{*} \circ \omega}=\varphi^{*} \circ \beta_{\omega}$, and Equation (4-9) follows.

\section{C. Examples.}

4C(a). Superfoliations of codimension $0+\varepsilon 1$. If $\mathscr{F}$ is a superfoliation of codimension $0+\varepsilon 1$ defined by an even 1-form $a \in \Omega^{1}(\mathcal{M})$, then there exists an odd 1-form $b \in \Omega^{1}(M)$ with $d_{M} a=-a \wedge b$.

By Proposition A.11, $H^{k}\left(\operatorname{Vect}(0,1)_{0}\right)=0$ if $k \notin\{0,1\}$, and $H^{1}\left(\operatorname{Vect}(0,1)_{0}\right) \simeq \mathbb{R}$. Moreover, $H^{1}\left(\operatorname{Vect}(0,1)_{0}\right)$ is generated by $H=\left[(\theta \partial / \partial \theta)^{*}\right]$. By Proposition 4.2, there exists a homomorphism $\omega: \bigwedge \operatorname{Vect}(0,1)^{*} \rightarrow \Omega(\mathcal{M})$ such that $\omega\left((\theta \partial / \partial \theta)^{*}\right)=$ $b$. By construction, we have $\varphi_{\mathcal{M F}}(H)=[p(b)]$. See [Laurent-Gengoux 2004] for additional details.

Example 4.8. Let $E \rightarrow S^{1}$ be a trivial 1-dimensional vector bundle. The supermanifold $S^{1,1}=\mathcal{M}\left(S^{1}, E\right)$, with base manifold $S^{1}$ and superalgebra of superfunctions $\Omega(M)=\Gamma\left(\bigwedge^{*} E\right)$, is called a supercircle. Let $x \in S^{1}$ be the even parameter and $\theta$ the odd parameter.

Let $t \in \mathbb{R}^{*}$ be a real number different from zero, and $\mathscr{F}_{t}$ the superfoliation of codimension $0+1 \varepsilon$ defined by the 1 -form $b_{t}=d \theta+t \theta d x$. Note that

$$
d_{S^{1,1}} b_{t}=t d x \wedge d \theta=(d \theta+t \theta d x) \wedge t d x .
$$

By the preceding discussion, we have

$$
\varphi_{S^{1,1, \mathscr{F}_{t}}}(H)=-[p(t d x)]=-t[d x] .
$$

For $t \neq 0$, we obtain a nonzero element of $H^{1}\left(S^{1}\right)$. We have thus constructed a supermanifold $M$ and a superfoliation $\mathscr{F}$ with $\varphi_{\mathcal{M}, \mathscr{F}}$ not zero.

4C(b). Superfoliations of codimension $1+\varepsilon 1$. Let $\mathscr{F}$ be a superfoliation of codimension $1+\varepsilon 1$ given by an odd 1 -form $\omega \in \Omega^{1}(\mathcal{M})$ and an even 1 -form $a \in \Omega^{1}(\mathcal{M})$.

There exist $b, c \in \Omega^{1}(\mathcal{M})$ with $d_{\mathcal{M}} a=\omega \wedge c-a \wedge b$. By Proposition 4.2, there is a DGA homomorphism $\omega_{\mathscr{F}}: \bigwedge \operatorname{Vect}(1,1)^{*} \rightarrow \Omega(\mathcal{M})$, defining the superfoliation $\mathscr{F}$, with $\omega_{\mathscr{F}}\left((\partial / \partial x)^{*}\right)=\omega$ and $\omega_{\mathscr{F}}\left((\theta \partial / \partial \theta)^{*}\right)=b$.

By Proposition A.12, $H^{k}\left(\operatorname{Vect}(n, m)_{0}\right)$ vanishes if $k \neq 3$, and $H^{3}\left(\operatorname{Vect}(n, m)_{0}\right)$ is generated the classes $H_{1}, H_{2}, H_{3}$ described by Equations (A-13), (A-14), and (A-15).

Proposition 4.9. Let $\mathscr{F}$ be a superfoliation of codimension $1+\varepsilon 1$ defined by the odd 1-form $\omega$ and the even 1-form a. Suppose $b$ is an odd 1-form and $c$ an even 1-form on $M$ satisfying $d_{\mathcal{M}} a=\omega \wedge c-a \wedge b$. 
(1) There exist $\alpha, \beta, \xi \in \Omega^{1}(M)$ on the base manifold $M$ such that

$$
\left\{\begin{array}{l}
d_{M} p(\omega)=p(\omega) \wedge \alpha, \\
d_{M} \alpha=p(\omega) \wedge \beta, \\
d_{M} p(b)=p(\omega) \wedge \xi
\end{array}\right.
$$

(2) We have

$$
\left\{\begin{array}{l}
\varphi_{\mathcal{M}, \mathscr{F}}\left(H_{1}\right)=[\beta \wedge \alpha \wedge p(\omega)], \\
\varphi_{\mathcal{M}, \mathscr{F}}\left(H_{2}\right)=[\xi \wedge \alpha \wedge p(\omega)], \\
\varphi_{\mathcal{M}, \mathscr{F}}\left(H_{3}\right)=[\xi \wedge p(b) \wedge p(\omega)] .
\end{array}\right.
$$

Proof. (1) Define $\alpha, \beta, \xi$ by

$$
\left\{\begin{array}{l}
\alpha=p \circ \omega_{\mathscr{F}}\left((x \partial / \partial x)^{*}\right) \\
\beta=p \circ \omega_{\mathscr{F}}\left(\left(\frac{1}{2} x^{2} \partial / \partial x\right)^{*}\right) \\
\xi=p \circ \omega_{\mathscr{F}}\left((x \theta \partial / \partial \theta)^{*}\right)
\end{array}\right.
$$

Applying $p \circ \omega_{\mathscr{F}}$ to the identity

$$
\partial\left(\frac{\partial}{\partial x}\right)^{*}=\left(\frac{\partial}{\partial x}\right)^{*} \wedge\left(x \frac{\partial}{\partial x}\right)^{*}-\left(\frac{\partial}{\partial \theta}\right)^{*} \wedge\left(\theta \frac{\partial}{\partial x}\right)^{*},
$$

and using Lemma 2.1, we obtain

$$
d_{M}(p(\omega))=p(\omega) \wedge \alpha .
$$

Applying $p \circ \omega_{\mathscr{F}}$ to the identity

$$
\partial\left(x \frac{\partial}{\partial x}\right)^{*}=\left(\frac{\partial}{\partial x}\right)^{*} \wedge\left(\frac{x^{2}}{2} \frac{\partial}{\partial x}\right)^{*}-\left(\frac{\partial}{\partial \theta}\right)^{*} \wedge\left(\theta x \frac{\partial}{\partial x}\right)^{*}-\left(x \frac{\partial}{\partial \theta}\right)^{*} \wedge\left(\theta \frac{\partial}{\partial x}\right)^{*},
$$

and using Lemma 2.1, we get

$$
d_{M}(\alpha)=p(\omega) \wedge \beta .
$$

Applying $p \circ \omega_{\mathscr{F}}$ to the identity

$$
\partial\left(\theta \frac{\partial}{\partial \theta}\right)^{*}=\left(\frac{\partial}{\partial x}\right)^{*} \wedge\left(x \theta \frac{\partial}{\partial \theta}\right)^{*}
$$

and using Lemma 2.1, we obtain

$$
d_{M}(p(b))=p(\omega) \wedge \xi .
$$

Thus, there exist 1-forms $\alpha, \beta, \xi$ satisfying (4-10). 
(2) We must now check that the cohomology classes

$$
[\beta \wedge \alpha \wedge p(\omega)],[\xi \wedge \alpha \wedge p(\omega)],[\xi \wedge p(b) \wedge p(\omega)] \in H^{3}(M)
$$

do not depend on the chosen 1-forms $\alpha, \beta, \xi$ that satisfy (4-10).

The 1-form $[\beta \wedge \alpha \wedge p(\omega)]$ is the Godbillon-Vey class of the codimension-1 foliation $F_{\mathscr{F}}$ constructed from $\mathscr{F}$ on the base manifold in Section $2 \mathrm{C}(\mathrm{a})$. This $F_{\mathscr{F}}$ is defined by $p(\omega)$. The fact that $[\beta \wedge \alpha \wedge p(\omega)]$ does not depend on the choice of $\alpha$ and $\beta$ was proved in [Godbillon and Vey 1971].

From the identity $\xi \wedge \alpha \wedge p(\omega)=-\left(d_{M} p(b)\right) \wedge \alpha=\xi \wedge d_{M} p(\omega)$ follows that $[\xi \wedge \alpha \wedge p(\omega)]$ does not depend on the 1-forms $\alpha, \beta, \xi$; while from the identity $\xi \wedge p(b) \wedge p(\omega)=-\left(d_{M} p(b)\right) \wedge p(b)$ follows that $[\xi \wedge \alpha \wedge p(\omega)]$ does not depend on the 1 -forms $\alpha, \beta, \xi$. This completes the proof.

Example 4.10. Let $M$ be a manifold and $F$ a foliation of codimension 1 on $M$, defined by $\omega \in \Omega^{1}(M)$ with $d_{M} \omega=\omega \wedge \alpha$. Assume moreover that the GodbillonVey class $-\left[\alpha \wedge d_{M} \alpha\right] \in H^{3}(M)$ is not zero.

Let $E \rightarrow M$ be the trivial 1-dimensional bundle $E=\mathbb{R} \times M$. Consider the supermanifold $\mathcal{M}$ with $\mathcal{O}(\mathcal{M})=\Gamma(\bigwedge E)$, and denote by $\theta$ the unique odd parameter corresponding to some constant section of $E$. There is a canonical embedding $I: \Omega^{1}(M) \rightarrow \Omega^{1}(\mathcal{M})$ given by the pull-back of the canonical projection $M \rightarrow M$. Of course, $p \circ I=\operatorname{Id}_{\Omega^{1}(M)}$.

Define a superfoliation of codimension $1+\varepsilon 1$ by the odd 1 -form $I(\omega)$ and the even 1-form $a=d \theta+\theta I(\alpha)$. We check that these 2-forms define a superfoliation. We have $d_{M} I(\omega)=I(\omega) \wedge I(\alpha)$ and

$$
d_{M} a=d \theta \wedge I(\alpha)+\theta d_{M} I(\alpha)=(d \theta+\theta I(\alpha)) \wedge I(\alpha)+\theta I\left(d_{M} \alpha\right) .
$$

Since $d_{M} \alpha=\omega \wedge \xi$ for some $\xi \in \Omega^{1}(M)$ [Godbillon and Vey 1971], we have

$$
d_{M} a=a \wedge I(\alpha)+I(\omega) \wedge \theta I(\xi) .
$$

Therefore, the pair $(a, I(\omega))$ defines a superfoliation of codimension $1+\varepsilon 1$.

Now, from (4-13) we see that $b=\alpha$ and $p(b)=\alpha$. By (4-11), we obtain

$$
\varphi_{\mathcal{M}, \mathscr{F}}\left(H_{1}\right)=\varphi_{\mathcal{M}, \mathscr{F}}\left(H_{2}\right)=\varphi_{\mathcal{M}, \mathscr{F}}\left(H_{3}\right)=-\left[\alpha \wedge d_{M} \alpha\right] \neq 0 .
$$

Example 4.11. We describe a superfoliation of codimension $1+\varepsilon 1$ with trivialized normal bundle such that $\varphi_{\mathcal{M}, \mathscr{H}}\left(H_{1}\right)=0$ and $\varphi_{\mathcal{M}, \mathscr{H}}\left(H_{3}\right) \neq 0$.

Consider the supermanifold given by the trivial 1-vector bundle $E$ over the 3dimensional torus $T^{3} \simeq\left(S^{1}\right)^{3}$. Let $x, y, z \in S^{1}$ be the coordinates of $T^{3}$, and let $\theta$ be the odd parameter corresponding to the constant section of $E$. Take $f(x), g(x)$ two smooth functions on $S^{1}$ with $\int_{S^{1}} W(f, g) \neq 0$, where $W$ is the Wronskian. 
We leave the reader to check that the odd 1-form $\omega=d x$ and the even 1-form

$$
a=d \theta+\theta(f(x) d y+g(x) d z)
$$

define a superfoliation of codimension $1+\varepsilon 1$. In this case, we can choose $b=$ $f(x) d y+g(x) d z$, and it is routine to check that

$$
\varphi_{\mathcal{M}, \mathscr{H}}\left(H_{3}\right)=[W(f, g) d x \wedge d y \wedge d z]
$$

Since $[W(f, g) d x \wedge d y \wedge d z]=\left(\int_{S^{1}} W(f, g)\right)[d x \wedge d y \wedge d z]$, it follows that $\varphi_{\mathcal{M}, \mathscr{H}}\left(H_{3}\right)$ is a nonzero class in de Rham cohomology. Since $p(\omega)=d x$ is a closed 1-form, $\varphi_{\mathcal{M}, \mathscr{H}}\left(H_{1}\right)=0$.

4C(c). Superfoliations of codimension $0+\varepsilon m$. According to Proposition A.10, we have $H^{*}\left(\operatorname{Vect}(0, m)_{0}\right) \simeq H^{*}\left(g l_{m}\right)$, where $H^{*}\left(g l_{m}\right)$ is the Chevalley-Eilenberg cohomology of the Lie algebra $g l_{m}$. In such a case, therefore, $\varphi_{\mathcal{M}, \mathscr{F}}$ is a map from $H^{*}\left(g l_{m}\right)$ to $H^{*}(M)$. Moreover, if $\omega$ is a DGA homomorphism defining the superfoliation $\mathscr{F}$, then $\varphi_{\mathcal{M}, \mathscr{F}}$ is given by

$$
d_{k}^{l} \rightarrow p\left(\omega\left(\left(\theta_{k} \partial / \partial \theta_{l}\right)^{*}\right)\right) \quad \text { for any } k, l=1, \ldots, m .
$$

Example 4.12. We compute this homomorphism in a particular case. The semidirect product $g l_{m} \ltimes \mathbb{R}^{m}$ of the Lie algebra $g l_{m}$ with $\mathbb{R}^{m}$ can be considered as a super-Lie algebra with even part $g l_{m}$ and odd part $\mathbb{R}^{m}$. Let $\varphi$ be the super-Lie group associated to this Lie algebra by Lie's third theorem (which is true for super-Lie algebras [Tuynman 2004]). Since $g l_{m} \subset g l_{m} \ltimes \mathbb{R}^{m}$, the Lie group $G L_{n}$ acts on the left on $\mathscr{G}$, and a superfoliation of codimension $0+\varepsilon m$ is given on $\mathscr{G}$ by this left action.

This superfoliation is defined by the left-invariant forms $\bar{a}_{1}, \ldots, \bar{a}_{m}$ associated to the canonical basis $a_{1}, \ldots, a_{m} \in\left(\mathbb{R}^{m}\right)^{*}$. Moreover, for any $k \in\{1, \ldots, m\}$, we have

$$
d_{\mathscr{c} g} \bar{a}_{k}=\sum_{l=1}^{m} \bar{a}_{l} \wedge \bar{d}_{k}^{l}
$$

where $\bar{d}_{k}^{l} \in T^{*}(\mathscr{G})$ are the left invariant 1-forms on $\mathscr{G}$ associated to the canonical basis $d_{k}^{l}$ of $g l(m)^{*}$.

By construction, therefore, there exists a homomorphism $\omega$ defining the superfoliation, such that

$$
\omega\left(\left(\theta_{k} \partial / \partial \theta_{l}\right)^{*}\right)=\bar{d}_{k}^{l} \quad \text { for any } k, l \in\{1, \ldots, m\} .
$$

By Equation (4-14), we obtain that, for any $H \in H^{*}\left(g l_{m}\right)$,

$$
\varphi_{\mathcal{M}, \mathscr{F}}(H)=\bar{H},
$$


where $\bar{H}$ is the class of the left-invariant form on $G L_{m}$ that corresponds to $H$. In other words, $\varphi_{\mathcal{M}, \mathscr{F}}$ is equal to the natural homomorphism $H^{*}\left(g l_{m}\right) \rightarrow H^{*}\left(G L_{m}\right)$.

It is well known that $H^{*}\left(G L_{m}\right) \simeq H^{*}(O(m))$, where $O(m)$ is the orthogonal group, and that $H^{3}(O(m)) \simeq \mathbb{R}$ and $H^{3}\left(g l_{m}\right) \simeq \mathbb{R}$ for $m \geq 3$. We leave the reader to check that the homomorphism $H^{3}\left(g l_{m}\right) \rightarrow H^{3}\left(G L_{m}\right)$ is not trivial. Therefore, we have proved the existence of nontrivial secondary characteristic classes for superfoliations of codimension $0+\varepsilon m$ with $m \geq 3$.

4D. Conclusion. We summarize the results of the preceding sections.

Theorem 4.13. For any supermanifold $M$ foliated by a superfoliation $\mathscr{F}$ of codimension $n+\varepsilon m$ with trivialized normal bundle, there exists a map $\varphi_{\mathcal{M}, \mathscr{F}}$ from $H^{*}\left(\operatorname{Vect}(n, m)_{0}\right)$ to $H^{*}(M)$ such that:

(1) $\varphi_{\mathcal{M}, \mathscr{F}}$ is a functor from the category of supermanifolds endowed with a superfoliation with trivialized normal bundle to the category of algebra homomorphisms from $H^{*}\left(\operatorname{Vect}(n, m)_{0}\right)$ to an algebra $A$;

(2) for $(n, m)=(0,1)$, or $(n, m)=(1,1)$, or $n=0$ and $m \geq 3$, there exists a supermanifold $\mu$ and a superfoliation $\mathscr{F}$ of codimension $n+\varepsilon m$ such that $\varphi_{\mathcal{M}, \mathscr{F}}$ is not the zero map;

(3) if $\mathcal{M}$ is an ordinary smooth manifold endowed with a foliation $F$ of codimension $n$ with trivialized normal bundle, then $\varphi_{F}$ reduces to the usual homomorphism of Bernstein, Bott, Fuchs, Haefliger and Rosenfeld [Bernstein and Rosenfeld 1973; Bott and Haefliger 1972; Fuchs 1986].

Proof. By "category of algebra homomorphisms from $H^{*}\left(\operatorname{Vect}(n, m)_{0}\right)$ to an algebra $A$ " we mean the category whose objects are algebra homomorphism from $H^{*}\left(\operatorname{Vect}(n, m)_{0}\right)$ to an algebra $A$, and whose arrows between objects

$$
\varphi_{A}: H^{*}\left(\operatorname{Vect}(n, m)_{0}\right) \rightarrow A \quad \text { and } \quad \varphi_{B}: H^{*}\left(\operatorname{Vect}(n, m)_{0}\right) \rightarrow B
$$

are homomorphisms $\varphi: A \rightarrow B$ such that $\varphi_{B}=\varphi \circ \varphi_{A}$. Conclusion (1) is now a paraphrase of Proposition 4.7.

Statement (2) follows from Examples 4.8, 4.10, and 4.12. Note that a more precise statement will be given in Remark 5.3.

It is proved in [Fuchs 1986, Section 3.2.B (page 231)] that, when we are given a foliation $F$ of codimension $n$ with trivialized normal bundle, the "classical" map of Bernstein, Bott, Fuchs, Haefliger and Rosenfeld is constructed by the passing to cohomology of a DGA homomorphism $\omega$ from $\left(\bigwedge \operatorname{Vect}(n)^{*}, \partial\right)$ to $\left(\Omega(M), d_{M}\right)$, with the foliation $F$ being defined by the 1 -forms $\omega\left(\left(\partial / \partial x_{i}\right)^{*}\right)$. This proves (3).

The functoriality of this construction allows us to say that the assignment $\mathscr{F} \mapsto$ $\varphi_{\mathscr{F}}(H)$, for any $H \in H^{*}\left(\operatorname{Vect}(n, m)_{0}\right)$, defines a secondary characteristic class of superfoliations with trivialized normal bundle. 
We have defined in Proposition 2.18 a way to construct from any flat trivial foliated connection $(M, F, E, \nabla)$ a supermanifold $M(M, E)$ whose base manifold is $M$, and a superfoliation $\mathscr{F}(M, F, E, \nabla)$ on $M$ with trivialized normal bundle. We call secondary characteristic classes of flat trivial foliated connections the secondary characteristic classes of this superfoliation. More precisely, we have associated a homomorphism from $H^{*}\left(\operatorname{Vect}(n, m)_{0}\right)$ to $H^{*}(M)$ to any flat trivial foliated connection on $M$.

Remark 4.14. One may ask whether, for any $H \in H^{*}\left(\operatorname{Vect}(n, m)_{0}\right)$, there is a superfoliation $\mathscr{F}$ and a supermanifold $\mathcal{M}$ with $\varphi_{\mathcal{M}, \mathscr{F}}(H) \neq 0$. This question remains open even in the case of foliations on smooth manifolds, and therefore we cannot hope to find a simple answer.

Remark 4.15. According to Section 2D, if the superfoliation $\mathscr{F}$ of codimension $n+\varepsilon m$ does not have a trivialized normal bundle, then we can replace it by a superfoliation $\operatorname{pr}^{*}(\mathscr{F})$ with trivialized normal bundle, over some $G L(n, m)$-principal bundle $\mathscr{P}_{\mathscr{F}} \rightarrow \mathcal{M}$. The map $\varphi_{\mathscr{P}_{\mathscr{F}}}, \mathrm{pr}^{*} \mathscr{F}_{\mathscr{F}}$ takes values in the cohomology of the base manifold of $\mathscr{P}_{\mathscr{F F}}$, which is a $G L(n) \times G L(m)$-principal bundle over $M$. Therefore, for any $H \in H^{*}(\operatorname{Vect}(n, m))$, we can construct secondary characteristic classes of superfoliation of codimension $n+\varepsilon m$ (not necessarily with trivialized normal bundle), but these characteristic classes have values in the cohomology of some $G L(n) \times G L(m)$-principal bundle over the base manifold $M$ of $M$. By Remark 2.21, this construction is functorial, that is, it behaves well with respect to pullbacks of superfoliations.

We now link this construction to the cohomology of $H(\operatorname{Vect}(n, m))$ and the Godbillon-Vey classes constructed in [Koszul 1988].

The homomorphism $\omega$ constructed in Proposition 4.2 induces a homomorphism $\varphi_{\omega}: H^{*}(\operatorname{Vect}(n, m)) \rightarrow H^{*}(\mathcal{M})$, where $H^{*}(\operatorname{Vect}(n, m))$ is the cohomology of the superalgebra $\operatorname{Vect}(n, m)$. The following diagram is commutative:

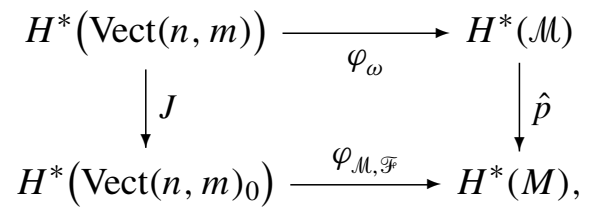

with $J: H^{*}\left(\operatorname{Vect}(n, m)_{0}\right) \rightarrow H^{*}(\operatorname{Vect}(n, m))$ given by the inclusion $\operatorname{Vect}(n, m)_{0} \rightarrow$ $\operatorname{Vect}(n, m)$, and $\hat{p}: H^{*}(\mathcal{M}) \rightarrow H^{*}(M)$ induced by $p$.

According to Batchelor's theorem, $\hat{p}$ is indeed an isomorphism, and thus $\varphi_{\omega}=$ $\hat{p}^{-1} \circ \varphi_{\mathcal{M}, \mathscr{F}} \circ J$ is independent of $\omega$. To emphasize that this homomorphism does not depend on $\omega$, we denote it by $\psi_{\mathcal{M}, \mathscr{F}}$. We cannot say that $\psi_{\mathcal{M}, \mathscr{F}}$ gives new secondary 
characteristic classes, since

$$
\psi_{M, \mathscr{F}}=\hat{p} \circ \varphi_{\mathcal{M}, \mathscr{F}} \circ J .
$$

In [Koszul 1988], a homomorphism from $H^{*}(\operatorname{Vect}(0, m))$ to $H^{*}(M)$ is associated to any superfoliation of codimension $n+\varepsilon m$ with trivialized normal bundle, on a supermanifold of dimension $n+\varepsilon m$. It is easy to check that, by construction, this homomorphism coincides with $\psi_{\mathcal{M}, \mathscr{F}}$. It defines classes of superfoliations of dimension $0+\varepsilon m$, called "Godbillon-Vey classes" by the author. By Equation (4-15), these Godbillon-Vey classes are among the classes we built in this article.

From Proposition A.11 and [Koszul 1988, Corollaire 1.2], it follows that $J$ : $H^{1}(\operatorname{Vect}(0,1)) \rightarrow H^{1}\left(\operatorname{Vect}(0,1)_{0}\right)$ is an isomorphism. This implies that the class constructed in Section 4C(a) is equal to the class constructed in [Koszul 1988, Exemple 1].

We summarize:

Proposition 4.16. (1) For any superfoliation F F codimension $n+\varepsilon m$ with trivialized normal bundle, the following diagram is commutative:

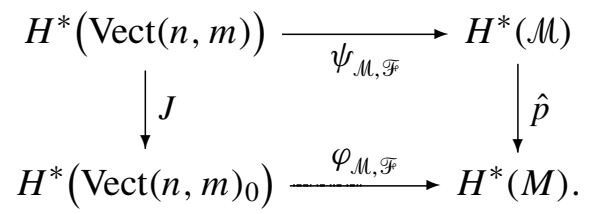

(2) The Godbillon-Vey classes constructed in [Koszul 1988] are among the secondary characteristic classes of superfoliation constructed above.

(3) In particular, for foliations of codimension $0+\varepsilon 1$, the secondary class constructed in Section 4C(a) coincides with the class constructed in [Koszul 1988, Exemple 1].

\section{Foliated flat vector bundles}

5A. Secondary characteristic classes on the base manifold. Let $F_{\mathscr{F}}$ be the codimension- $n$ foliation induced by $\mathscr{F}$ on $M$, as in Lemma 2.12. Since $F$ has a trivialized normal bundle, according to Theorem 4.13 to any $H \in H^{*}(\operatorname{Vect}(n))$ is associated an element $K \in H^{*}(M)$ by the "classical" construction of Bernstein, Bott, Fuchs, Haefliger and Rosenfeld [Bernstein and Rosenfeld 1973; Bott and Haefliger 1972; Fuchs 1986]. We would like to investigate the relation between this construction and our construction.

There is a natural inclusion $i$ from $\operatorname{Vect}(n)^{*}$ into $\operatorname{Vect}(n, m)_{0}^{*}$, obtained by considering an element $\left(x_{1}^{i_{1}} \ldots x_{n}^{i_{n}} \partial / \partial x_{a}\right)^{*} \in \operatorname{Vect}(n)^{*}$ as an element of $\operatorname{Vect}(n, m)_{0}^{*}$.

Lemma 5.1. The inclusion $i$ is a DGA homomorphism, and induces a map from $H^{*}(\operatorname{Vect}(n))$ to $H^{*}\left(\operatorname{Vect}(n, m)_{0}\right)$. 
Proof. The family

$$
\left\{\begin{array}{l}
x_{1}^{i_{1}} \ldots x_{n}^{i_{n}} \theta_{1}^{j_{1}} \ldots \theta_{m}^{j_{m}} \frac{\partial}{\partial x_{i}} \\
\text { and } \\
x_{1}^{i_{1}} \ldots x_{n}^{i_{n}} \theta_{1}^{j_{1}} \ldots \theta_{m}^{j_{m}} \frac{\partial}{\partial \theta_{j}}
\end{array}\right.
$$$$
i_{1}, \ldots, i_{n} \in \mathbb{N}
$$$$
\begin{aligned}
& i_{1}, \ldots, i_{n} \in \mathbb{N} \\
& j_{1}, \ldots, j_{m} \in\{0,1\} \text { with } \sum_{k=1}^{n} j_{k} \text { even, } \\
& \text { and } i \in\{1, \ldots, n\}
\end{aligned}
$$$$
i_{1}, \ldots, i_{n} \in \mathbb{N}
$$$$
\begin{aligned}
& j_{1}, \ldots, j_{m} \in\{0,1\} \text { with } \sum_{k=1}^{m} j_{k} \text { odd, } \\
& \text { and } j \in\{1, \ldots, m\},
\end{aligned}
$$

forms a basis of $\operatorname{Vect}(n, m)_{0}$.

The $\theta$-degree $\left(h_{i_{1}} \ldots h_{i_{k}} \partial / \partial h_{i}\right) \in \operatorname{Vect}(n, m)_{0}$ is the number of integers greater or equal to $n+1$ in the list $i_{1}, \ldots, i_{k}, i$. For example, the $\theta$-degree of $x_{1} \theta_{1} \partial / \partial \theta_{2}$ or $x_{3} \theta_{2} \theta_{1} \partial / \partial x_{2}$ is 2 , and the $\theta$-degree of $x_{1}^{3} \partial / \partial x_{1}$ is 0 .

If we enumerate the basis as described in (5-1), then the structure constant $\Gamma_{i, j}^{k}$ is equal to zero if the index $k$ corresponds to an element of $\theta$-degree 0 and one of the indices $i$ or $j$ corresponds to an element of nonzero $\theta$-degree. Moreover, if $i, j, k$ correspond to elements of the basis (5-1) with a vanishing $\theta$-degree, then the structure constant $\Gamma_{i, j}^{k}$ is equal to the corresponding structure constant in $\operatorname{Vect}(n)$. By the definition of the Chevalley-Eilenberg differential, this implies that $i\left(\bigwedge \operatorname{Vect}(n)^{*}\right)$ is stable under $\partial_{0}$, and that $i$ is a DGA homomorphism.

Proposition 5.2. Take $H \in H^{*}\left(\operatorname{Vect}(n)^{*}\right)$ and $H^{\prime}=i(H) \in H^{*}\left(\operatorname{Vect}(n, m)_{0}\right)$. One has

$$
\varphi_{M, F}(H)=\varphi_{\mathcal{M}, \mathscr{F}}\left(H^{\prime}\right) .
$$

Proof. If $\omega: \bigwedge \operatorname{Vect}(n, m) \rightarrow \Omega(M)$ is a DGA homomorphism that defines $\mathscr{F}$, then $p \circ \omega \circ i: \wedge \operatorname{Vect}(n) \rightarrow \Omega(M)$ is a DGA homomorphism that defines $F$. The diagram

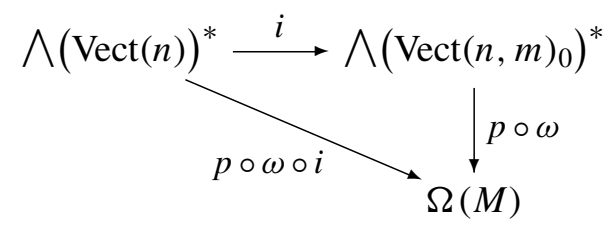

is commutative. As a consequence, we have the commutative diagram

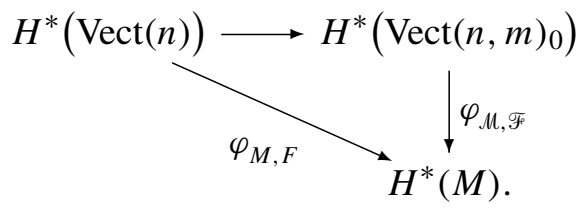

Remark 5.3. The nontrivial secondary classes in Section $4 \mathrm{C}$ are not secondary classes of the induced foliation on the base manifold. We could therefore replace (2) in Theorem 4.13 by a more precise statement: 
Let $S$ be a supplement of $H^{*}(\operatorname{Vect}(n))$ in $H^{*}\left(\operatorname{Vect}(n, m)_{0}\right)$. For $(n, m)=(0,1)$, or $(n, m)=(1,1)$, or $n=0$ and $m \geq 3$, there exists a supermanifold $M$ and $a$ superfoliation $\mathscr{F}$ of codimension $n+\varepsilon m$ such that the restriction of $\varphi_{\mathcal{M}, \mathscr{F}}$ to $S$ is not the zero map.

5B. Secondary characteristic classes of foliated connections. Let $\mathscr{F}$ be a superfoliation of codimension $n+\varepsilon m$ with trivialized normal bundle, defined by odd 1 -forms $\omega_{1}, \ldots, \omega_{n}$ and even 1 -forms $a_{1}, \ldots, a_{m}$. It is convenient to rename these forms $b_{1}, \ldots, b_{n+m}$, where $b_{i}=\omega_{i}$ for all $i \in\{1, \ldots, n\}$ and $b_{n+j}=a_{j}$ for all $j \in\{1, \ldots, m\}$. In fact, we will in general use both notations at the same time. Let $\left(M, F_{\mathscr{F}}, E_{\mathscr{F}}, \nabla^{\mathscr{F}}\right)$ be the trivial flat foliated connection associated to the superfoliation $\mathscr{F}$, as constructed in Section 2C.

Theorem 5.4. The homomorphism $\varphi_{\mathcal{M}, \mathscr{F}}: H^{*}\left(\operatorname{Vect}(n, m)_{0}\right) \rightarrow H^{*}(M)$ is completely determined by the flat trivial foliated connection $\left(M, F_{\mathscr{F}}, E_{\mathscr{F}}, \nabla^{\mathscr{F}}\right)$.

In other words, the theory of secondary characteristic classes of superfoliations can indeed be considered as a theory of secondary characteristic classes of flat foliated connections.

Proof. Let $\left(b_{i}^{j}\right)_{i, j \in\{1, \ldots, n+m\}}$ be homogeneous 1-forms satisfying the relation

$$
d_{M} b_{i}=(-1)^{|j|} \sum_{j=1}^{n+m} b_{j} \wedge b_{i}^{j}
$$

We divide the proof into four steps.

Step 1: $\varphi_{\mathcal{M}, \mathscr{F}}$ depends only on $\left(p\left(b_{i}^{j}\right)\right)_{i, j=1, \ldots, n}$ and $\left(p\left(b_{k}^{l}\right)\right)_{k, l \in\{n+1, \ldots, n+m\}}$.

By Lemma 4.6, the secondary characteristic classes depend only on the 1-forms $\left(p\left(b_{i}^{j}\right)\right)_{i, j \in\{1, \ldots, n\}}$ and $\left(p\left(b_{k}^{l}\right)\right)_{i, j \in\{n+1, \ldots, n+m\}}$. Moreover, any family of 1-forms $\left(\tilde{b}_{i}^{j}\right)_{i, j \in\{1, \ldots, n+m\}}$ such that (5-2) holds for some 1-forms $\tilde{b}_{1}, \ldots, \tilde{b}_{n+m}$ that define the same superfoliation $\mathscr{F}_{\mathscr{F}}$ will define the same homomorphism $\varphi_{\mathcal{M}, \mathscr{F}}$ by Proposition 4.5.

Step 2: $\varphi_{\mathcal{M}, \mathscr{F}}$ depends only on $F_{\mathscr{F}}$ and $\left(p\left(b_{k}^{l}\right)\right)_{k, l \in\{n+1, \ldots, n+m\}}$.

After applying $p$ to (5-2) and taking $i \in\{1, \ldots, n\}$, we see that the 1 -forms $\left(p\left(b_{i}^{j}\right)\right)_{i, j \in\{1, \ldots, n\}}$ satisfy

$$
d_{M}\left(p\left(\omega_{i}\right)\right)=\sum_{j=1}^{n} p\left(\omega_{j}\right) \wedge p\left(b_{i}^{j}\right) .
$$

By Remark 2.13, the 1 -forms $p\left(\omega_{i}\right), i=1, \ldots, n$, define the foliation $F_{\mathscr{F}}$. For any other choice of 1-forms $\tilde{\omega}_{1}, \ldots, \tilde{\omega}_{n} \in \Omega^{1}(M)$ and any 1-forms $\tilde{c}_{i}^{j} \in \Omega^{1}(M), i, j \in$ $\{1, \ldots, n\}$, with $d_{M} \tilde{\omega}_{i}=\sum_{j=1}^{n} \tilde{\omega}_{j} \wedge c_{i}^{j}$, there exist 1 -forms $\tilde{b}_{1}, \ldots, \tilde{b}_{n+m} \in \Omega^{1}(\mathcal{M})$ 
defining $\mathscr{F}_{F}$, and 1-forms $\tilde{b}_{i}^{j} \in \Omega_{1}(M), i, j \in\{1, \ldots, n+m\}$, satisfying (5-2) such that, for all $i, j \in\{1, \ldots, n\}$, the identity $p\left(b_{i}^{j}\right)=c_{i}^{j}$ holds. As a consequence, $\varphi_{\mathcal{M}, \mathscr{F}}$ is entirely determined by $F_{\mathscr{F}}$ and $\left(p\left(b_{k}^{l}\right)\right)_{k, l \in\{n+1, \ldots, n+m\}}$

Step 3: $\varphi_{\mathcal{M}, \mathscr{F}}$ depends only on $F_{\mathscr{F}}$ and on the restriction of $\left(p\left(b_{k}^{l}\right)\right)_{k, l \in\{n+1, \ldots, n+m\}}$ to the tangent space of the leaves of $F_{\mathscr{F}}$.

For $k \in\{n+1, \ldots, n+m\}$, Equation (5-2) can be rewritten

$$
d_{M} a_{k-n}=\sum_{i=1}^{n} \omega_{i} \wedge b_{k}^{i}-\sum_{l=n+1}^{n+m} a_{l} \wedge b_{k}^{l} .
$$

As consequence, for any even superfunctions $f_{k, j}^{l}$ with $k, j \in\{1, \ldots, m\}$ and $l \in$ $\{1, \ldots, n\}$,

$$
d_{M} a_{k-n}=\sum_{i=1}^{n} \omega_{i} \wedge\left(b_{k}^{i}-\sum_{c=1}^{m} f_{i}^{k, c} a_{c}\right)-\sum_{l=n+1}^{n+m} a_{l} \wedge\left(b_{k}^{l}-\sum_{c=1}^{n} f_{c}^{k, l} \omega_{c}\right) .
$$

We have therefore

where

$$
d_{M} a_{k-n}=\sum_{i=1}^{n} \omega_{i} \wedge \tilde{b}_{k}^{i}-\sum_{l=n+1}^{n+m} a_{l} \wedge \tilde{b}_{k}^{l}
$$

$$
\tilde{b}_{k}^{i}=b_{k}^{i}-\sum_{c=n+1}^{n+m} f_{i}^{k, c} a_{c} \quad \text { and } \quad \tilde{b}_{k}^{l}=b_{k}^{l}-\sum_{c=1}^{n} f_{c}^{k, l} \omega_{c} .
$$

Consequently, we can add to the 1 -forms $\left(b_{k}^{l}\right)_{k, l \in\{n+1, \ldots, n+m\}}$ any linear combination of the 1 -forms $\omega_{1}, \ldots, \omega_{n}$ without modifying $\varphi_{\mathcal{M}, \mathscr{F}}$.

Applying $p$ to (5-3), we obtain

$$
p\left(\tilde{b}_{l}^{k}\right)=p\left(b_{l}^{k}\right)-\sum_{c=1}^{n} \operatorname{Re}\left(f_{c}^{k, l}\right) p\left(\omega_{c}\right)
$$

Therefore, we can add to the 1 -forms $\left(p\left(b_{k}^{l}\right)\right)_{k, l=n+1, \ldots, n+m}$ any linear combination of the 1-forms $p\left(\omega_{1}\right), \ldots, p\left(\omega_{n}\right)$ without modifying $\varphi_{\mathcal{M}, \mathscr{F}}$. This implies that $\varphi_{\mathcal{M}, \mathscr{F}}$ depends only on the restriction of $\left(p\left(b_{k}^{l}\right)\right)_{k, l \in\{n+1, \ldots, n+m\}}$ to the tangent spaces of the leaves of $F_{\mathscr{F}}$.

Step 4: The restriction of $\left(p\left(b_{k}^{l}\right)\right)_{k, l \in\{n+1, \ldots, n+m\}}$ to the tangent space of the leaves of $F_{\mathscr{F}}$ depends only on $E_{\mathscr{F}}$ and $\nabla^{\mathscr{F}}$.

Let $X$ be a vector field tangent to the leaves of $F_{\mathscr{F}}$, and $\mathscr{X} \in\left(\mathscr{X}_{\mathscr{F}}\right)_{0}$ an even supervector field tangent to $\mathscr{F}$ such that $\Pi(\mathscr{X})=X$. One has, for any $k \in\{n+1, \ldots, n+m\}$,

$$
\begin{aligned}
\nabla_{X}^{\mathscr{F}}\left(\rho\left(a_{k-n}\right)\right) & =\rho\left(L_{\mathscr{X}} a_{k-n}\right)=\rho\left(\iota_{\mathscr{C}} d_{M} a_{k-n}\right)=\rho\left(\iota_{\mathscr{X}} \sum_{j=1}^{n+m}(-1)^{|j|} b_{j} \wedge b_{k}^{j}\right) \\
& =\rho\left(\iota_{\mathscr{X}} \sum_{j=1}^{n} \omega_{j} \wedge b_{k}^{j}-\sum_{l=n+1}^{n+m} a_{j} \wedge b_{k}^{l}\right) .
\end{aligned}
$$


By the definition of the map $\rho$, one has $\rho\left(\left(\iota_{\mathscr{L}} \omega_{j}\right) b_{n+i}^{j}\right)=0$ for any $j \in\{1, \ldots, n+m\}$ and $\rho\left(\iota_{\mathscr{L}}\left(\omega_{j} \wedge b_{n+i}^{j}\right)\right)=0$ for any $j \in\{1, \ldots, n\}$. Therefore,

$$
\nabla_{X}^{\mathscr{F}}\left(\rho\left(a_{k-n}\right)\right)=-\rho\left(\sum_{j=1}^{m}\left(\iota_{\mathscr{X}} a_{j} \wedge b_{n+i}^{n+j}\right)\right) .
$$

By Equation (2-1), we obtain

$$
\nabla_{X}^{\mathscr{F}}\left(\rho\left(a_{k-n}\right)\right)=-\sum_{l=n+1}^{n+m}\left(\operatorname{Re}\left(l_{\mathscr{L}} b_{k}^{l}\right)\right) \rho\left(a_{j}\right) .
$$

By (2-4), the identity $\operatorname{Re}\left(\iota_{\mathscr{L}} b_{k}^{l}\right)=\iota_{X} p\left(b_{k}^{l}\right)$ holds, and thus

$$
\nabla_{X}^{\mathscr{F}}\left(\rho\left(a_{k-n}\right)\right)=-\sum_{l=n+1}^{n+m}\left(\iota_{X} p\left(b_{k}^{l}\right)\right) \rho\left(a_{l}\right) .
$$

The restrictions of the 1 -forms $\left(p\left(b_{n+i}^{n+j}\right)\right)_{i, j \in\{1, \ldots, m\}}$ to the tangent space of each leaf of $F_{\mathscr{F}}$ are therefore completely determined by the connection $\nabla^{\mathscr{F}}$. This completes the proof.

Remark 5.5. We have associated secondary characteristic classes to a flat trivial foliated vector bundle. In particular, we have associated characteristic classes to any $S O(n)$-bundle over a manifold $M$, endowed with a flat foliated connection in the sense of [Kamber and Tondeur 1975]. In [Kamber and Tondeur 1974] or [Kamber and Tondeur 1975], characteristic classes are also associated to such objects. It should be interesting to investigate the relation with this construction, in particular with the map (4.4) from [Kamber and Tondeur 1974].

Remark 5.6. Before ending this section, we have to point out the relation between this approach and the theory of $\Gamma$-structures [Bott and Haefliger 1972]. A foliation on a supermanifold $M$ with base manifold $M$ can be defined by a family $\left\{f_{i}\right\}_{i \in I}$ of local submersions onto $\mathbb{R}^{n, m}$, defined on an open covering $\left\{U_{i}\right\}_{i \in I}$ of $M$. For any two submersions $f_{i}$ and $f_{j}$, there exists $\varphi_{i}^{j}$ in $\operatorname{Diff}\left(\mathbb{R}^{n, m}\right)$ such that $f_{i}=\varphi_{i}^{j} \circ f_{j}$, where $\operatorname{Diff}\left(\mathbb{R}^{n, m}\right)$ is the pseudogroup of local diffeomorphisms of the supermanifold $\mathbb{R}^{n, m}$. This is (almost) the definition of a $\operatorname{Vect}(n, m)$-structure from [Bott and Haefliger 1972]; the only difference is that $\Gamma$ is not a subspace of local diffeomorphisms of some vector space. As a consequence, it should be possible to obtain again most constructions of the present paper by generalizing the results of [Bott and Haefliger 1972] to this case; note that the Lie algebra of $\operatorname{Diff}\left(\mathbb{R}^{n, m}\right)$ is precisely $\operatorname{Vect}(n, m)_{0}$.

\section{Appendix A. The cohomology of $\operatorname{Vect}(n, m)_{0}$}

We prove Theorem 3.7 and, as an application, compute $H^{*}\left(\operatorname{Vect}(n, m)_{0}\right)$ in some particular cases. First, we will need some technical results about representations 
of the Lie algebra $g l_{n} \oplus g l_{m}$. The methods of this section are mainly inspired by [Astashkevich and Fuchs 1993; Fuchs 1986].

For any vector space $V$ and any $k \in \mathbb{N}$, we denote by $V^{\otimes k}$ the tensor-product of $k$ copies of $V$. For any family of vector spaces $V_{1}, \ldots, V_{k}$, we denote by $\bigotimes_{i=1}^{k} V_{i}$ the tensor product $V_{1} \otimes V_{2} \otimes \ldots \otimes V_{k}$. We denote by $S^{k}(V)$ the space of elements of degree $k$ in the symmetric algebra of $V$.

A1. Some results about representations of $\boldsymbol{g} \boldsymbol{l}_{\boldsymbol{n}} \oplus \boldsymbol{g} \boldsymbol{l}_{\boldsymbol{m}}$. Let $V$ and $W$ be vector spaces of dimension $n$ and $m$, respectively. Let $g l_{n}$ and $g l_{m}$ be the Lie algebras of linear endomorphism of $V$ and $W$, respectively. The Lie algebras $g l_{n}$ and $g l_{m}$ act on $V$ and $W$, respectively, by $g \cdot v=g(v)$ and $h \cdot w=h(w)$, where $v \in V, g \in g l_{n}$, $w \in W, h \in g l_{m}$. Moreover, the dual spaces $V^{*}$ and $W^{*}$ are $g l_{n^{-}}$and $g l_{m}$-modules, respectively, with actions

$$
g \cdot v^{\prime}=(-g)^{*}\left(v^{\prime}\right) \quad \text { and } \quad h \cdot w^{\prime}=(-h)^{*}(w),
$$

where $g^{*}$ and $h^{*}$ are the dual endomorphisms and $v^{\prime} \in V^{*}, w^{\prime} \in W^{*}$. These actions extend naturally to an action of the Lie algebra $g l_{n} \oplus g l_{m}$ on

$$
E_{p, q}^{k, l}=V^{\otimes k} \otimes\left(V^{*}\right)^{\otimes l} \otimes W^{\otimes p} \otimes\left(W^{*}\right)^{\otimes q} .
$$

We give some lemmas about the representations of $g l_{n} \oplus g l_{m}$. It is well known (see [Fuchs 1986, Theorem 2.1.4] or [Howe 1989]) that, for any vector space $V$,

$$
\left\{\begin{array}{l}
\left(V^{\otimes k} \otimes\left(V^{*}\right)^{\otimes l}\right)^{g l_{n}}=0 \quad \text { if } k \neq l, \\
\left(V^{\otimes k} \otimes\left(V^{*}\right)^{\otimes k}\right)^{g l_{n}}=\bigoplus_{\sigma \in \Sigma_{k}} a_{\sigma} \sum_{i_{1}=1}^{n} \ldots \sum_{i_{k}=1}^{n} x_{i_{1}} \otimes \cdots \otimes x_{i_{n}} \otimes y_{i_{\sigma(1)}} \otimes \cdots \otimes y_{i_{\sigma(n)}},
\end{array}\right.
$$

where $a_{\sigma} \in \mathbb{R}, x_{1}, \ldots, x_{n} \in V$ is a basis of $V$, and $y_{1}, \ldots, y_{n} \in V^{*}$ is the dual basis. The following is an obvious generalization of this result:

Lemma A.1. Let $\left\{x_{i} \mid i=1, \ldots, n\right\}$ be a basis of $V$ and $\left\{y_{i} \in V^{*} \mid i=1, \ldots, n\right\}$ the dual basis. Let $\left\{\zeta_{i} \mid i=1, \ldots m\right\}$ be a basis of $W$ and $\left\{\eta_{i} \in W^{*} \mid i=1, \ldots, m\right\}$ the dual basis.

(1) If $k \neq l$ or $p \neq q$, then $\left(E_{p, q}^{k, l}\right)^{g l_{n} \oplus g l_{m}}=0$

(2) If $k=l$ and $p=q$, the space $\left(E_{p, p}^{k, k}\right)^{g l_{n} \oplus g l_{m}}$ is generated by the elements

$$
\begin{aligned}
g_{\sigma, \tau}= & \sum_{s_{1}=1}^{n} \ldots \sum_{s_{k}=1}^{n} \sum_{s_{1}^{\prime}=1}^{m} \ldots \sum_{s_{p}^{\prime}=1}^{m} \\
& x_{s_{1}} \otimes \ldots \otimes x_{s_{k}} \otimes y_{s_{\sigma(1)}} \otimes \ldots \otimes y_{s_{\sigma(k)}} \otimes \zeta_{s_{1}^{\prime}} \otimes \ldots \otimes \zeta_{s_{p}^{\prime}} \otimes \eta_{s_{\tau(1)}^{\prime}} \otimes \ldots \otimes \eta_{s_{\tau(p)}^{\prime}},
\end{aligned}
$$

where $\sigma$ is a permutation of $\{1, \ldots, k\}$ and $\tau$ a permutation of $\{1, \ldots, p\}$. 
Let $H$ be a $g l_{n} \oplus g l_{m}$-submodule of $V^{\otimes k} \otimes\left(V^{*}\right)^{\otimes m} \otimes W^{\otimes p} \otimes\left(W^{*}\right)^{\otimes l}$. Let $G$ be the $g l_{n} \oplus g l_{m}$-module

$$
G:=E_{p, q}^{k, l} / H
$$

Such a $g l_{n} \oplus g l_{m}$-module is said to be of type $I$.

Lemma A.2. Let $\pi$ be the projection of $E_{p, q}^{k, l}$ onto $G$. One has:

$$
G^{g l_{n} \oplus g l_{m}}=\pi\left(E_{p, q}^{k, l}\right)^{g l_{n} \oplus g l_{m}} .
$$

Proof. Both spaces reduce to 0 if $k \neq l$ or if $p \neq q$. If $k=l$ and $p=q$, the center of $g l_{n} \oplus g l_{m}$ acts trivially, and then both $G$ and $E_{p, q}^{k, l}$ are $s l_{n} \oplus s l_{m}$-modules satisfying

$$
G^{s l_{n} \oplus s l_{m}}=G^{g l_{n} \oplus g l_{m}}, \quad\left(E_{p, p}^{k, k}\right)^{s l_{n} \oplus s l_{m}}=\left(E_{p, p}^{k, k}\right)^{g l_{n} \oplus g l_{m}} .
$$

Since $s l_{n} \oplus s l_{m}$ is a semisimple Lie algebra, any finite-dimensional $s l_{n} \oplus s l_{m}$-module is the direct sum of the $s l_{n} \oplus s l_{m}$-submodule of invariants with the $s l_{n} \oplus s l_{m}$ submodule of coinvariants. In particular, we have the decompositions

$$
\left\{\begin{array}{l}
G=\left(s l_{n} \oplus s l_{m}\right) \cdot G \oplus G^{s l_{n} \oplus s l_{m}} \\
E_{p, p}^{k, k}=\left(s l_{n} \oplus s l_{m}\right) \cdot E_{p, p}^{k, k} \oplus\left(E_{p, p}^{k, k}\right) s l_{n} \oplus s l_{m}
\end{array}\right.
$$

Since $\pi$ is a Lie algebra morphism,

$$
\left\{\begin{array}{l}
\pi\left(\left(E_{p, p}^{k, k}\right)^{s l_{n} \times s l_{m}}\right) \subset G^{s l_{n} \oplus s l_{m}} \\
\pi\left(\left(s l_{n} \oplus s l_{m}\right) . E_{p, p}^{k, k}\right) \subset\left(s l_{n} \oplus s l_{m}\right) . G
\end{array}\right.
$$

Since $\pi$ is onto, we must have

$$
\left\{\begin{array}{l}
\pi\left(\left(E_{p, p}^{k, k}\right)^{s l_{n} \oplus s l_{m}}\right)=G^{s l_{n} \oplus s l_{m}} \\
\left(s l_{n} \oplus s l_{m}\right) \cdot \pi\left(E_{p, p}^{k, k}\right)=\left(s l_{n} \oplus s l_{m}\right) . G
\end{array}\right.
$$

The conclusion thus follows from (A-2).

We now introduce the $g l_{n} \oplus g l_{m}$-modules that we will study. (In the sequel, we will often simply say "module" for a $g l_{n} \oplus g l_{m}$-module.)

Definition A.3. Consider a family $L_{1}=\left\{\left(\mu_{a}, v_{a}\right) \mid a=1, \ldots, K\right\}$ of pairs of nonnegative integers such that $\mu_{a}+v_{a} \geq 2$ and $v_{a}$ is even. Consider a family $L_{2}=\left\{\left(p_{b}, q_{b}\right) \mid b=1 \ldots, K^{\prime}\right\}$ of pairs of nonnegative integers such that $p_{b}+q_{b} \geq 2$ and $q_{b}$ is odd. Let $L \in \mathbb{N}$ and let $\mathscr{L}=L_{1} \sqcup L_{2} \sqcup\{L\}$. We associate to $\mathscr{L}$ the $g l_{n} \oplus g l_{m}$ module

$$
G_{\mathscr{L}}=\bigwedge_{a=1}^{K}\left(S^{\mu_{a}}(V) \otimes \bigwedge^{v_{a}} W \otimes V^{*}\right) \wedge \bigwedge_{b=1}^{K^{\prime}}\left(S^{p_{b}}(V) \otimes \bigwedge^{q_{b}} W \otimes W^{*}\right) \wedge \bigwedge^{L} V^{*} .
$$

Such a module is called a module of type II. 
Our goal for now is to describe $\left(G_{\mathscr{L}}\right)^{g l_{n} \oplus g l_{m}}$; see Section A2 for motivation.

We describe a natural projection

$$
\pi_{\mathscr{L}}: Q \rightarrow G_{F}
$$

where $Q=E_{\mathfrak{n}, K^{\prime}}^{\mathfrak{m}, K+L}$, with $\mathfrak{m}=\sum_{a=1}^{K} \mu_{a}+\sum_{b=1}^{K^{\prime}} p_{b}$ and $\mathfrak{n}=\sum_{a=1}^{K} v_{a}+\sum_{b=1}^{K^{\prime}} q_{b}$.

First, we can construct an isomorphism $\psi$ of $g l_{n} \oplus g l_{m}$-module from $Q$ to the module

$$
Q_{\mathscr{L}}=\bigotimes_{a=1}^{K}\left(V^{\otimes \mu_{a}} \otimes W^{\otimes \mu_{a}} \otimes V^{*}\right) \otimes \bigotimes_{b=1}^{K^{\prime}}\left(V^{\otimes p_{b}} \otimes W^{\otimes q_{b}} \otimes W^{*}\right) \otimes\left(V^{*}\right)^{\otimes L}
$$

obtained by permuting terms in the tensor product defining $Q$, according to the rule: allow permutation of the tensor product of two elements if and only if they do not belong to the same vector space. With this restriction, there is only one natural isomorphism $\psi$ from $Q$ to $Q_{\mathscr{L}}$.

Second, there is a natural projection

$$
\pi^{\prime}: \bigotimes_{a=1}^{K}\left(V^{\otimes \mu_{a}} \otimes W^{\otimes v_{a}} \otimes V^{*}\right) \otimes\left(\bigotimes_{b=1}^{K^{\prime}} V^{\otimes p_{b}} \otimes W^{\otimes q_{b}} \otimes W^{*}\right) \otimes\left(V^{*}\right)^{\otimes L} \longrightarrow G_{\mathscr{L}}
$$

constructed by taking the appropriate symmerizations and skew-symmetrizations. Define $\pi_{\mathscr{L}}$ as $\pi_{\mathscr{L}}=\pi^{\prime} \circ \psi$.

Proposition A.4. (1) If $\sum_{a=1}^{K} \mu_{a}+\sum_{b=1}^{K^{\prime}} p_{b} \neq K+L$ or if $\sum_{a=1}^{K} v_{a}+\sum_{b=1}^{K^{\prime}} q_{b} \neq$ $K^{\prime}$, then $G_{\mathscr{L}}^{g l_{n} \oplus g l_{m}}=0$.

(2) If there exists $a \in\{1, \ldots, K\}$ with $v_{a} \neq 0$, or if there exists $b \in\left\{1, \ldots, K^{\prime}\right\}$ with $q_{b} \neq 1$, then $G_{\mathscr{L}}^{g l_{n} \oplus g l_{m}}=0$.

Proof. Since $G_{\mathscr{L}}$ is a module of type I, Lemma A.1(1) and Lemma A.2 immediately imply statement (1). The identity $\sum_{a=1}^{K} v_{a}+\sum_{b=1}^{K^{\prime}} q_{b}=K^{\prime}$ implies statement (2), since $q_{b}$ is odd for $b=1, \ldots, K$, and $v_{a}$ is even for $a=1, \ldots, K$, and since all these numbers are nonnegative.

From now on we assume that $q_{1}=\cdots=q_{K^{\prime}}=1, v_{1}=\cdots=v_{K}=0$, and

$$
\sum_{a=1}^{K} \mu_{a}+\sum_{b=1}^{K^{\prime}} p_{b}=K+L
$$

Write $H=\sum_{a=1}^{K} \mu_{a}$.

The map $\pi_{M}$ can now be easily described: for all $s_{1}, \ldots, s_{K+L} \in\{1, \ldots, n\}$, $t_{1}, \ldots, t_{K^{\prime}} \in\{1, \ldots, m\}, s_{1}^{\prime}, \ldots, s_{K^{\prime}}^{\prime} \in\{1, \ldots, m\}$, and $t_{1}^{\prime}, \ldots, t_{K^{\prime}}^{\prime} \in\{1, \ldots, m\}$, we have 
(A-4)

$$
\begin{aligned}
& \pi_{\mathscr{L}}\left(x_{s_{1}} \otimes \cdots x_{s_{K+L}} \cdot t_{s_{1}} \otimes \cdots y_{t_{K^{\prime}}^{\prime}} \zeta_{s_{1}^{\prime}} \cdots \cdots \zeta_{s_{K^{\prime}}^{\prime}} \cdot \eta_{t_{1}^{\prime}} \cdots \cdots \eta_{t_{K^{\prime}}^{\prime}}\right) \\
& =\bigwedge_{i=0}^{K} R\left(s_{\mu_{1}+\cdots+\mu_{i-1}+1}, \ldots, s_{\mu_{1}+\cdots+\mu_{i}}\right) \\
& \left(x_{s_{\mu_{1}+\cdots+\mu_{i}+1}} \ldots x_{s_{\mu_{1}+\cdots+\mu_{i+1}}} \cdot y_{t_{i}}\right) \\
& \wedge \bigwedge_{j=0}^{K^{\prime}} R\left(s_{H+p_{1}+\cdots+p_{j-1}+1}, \ldots, s_{H+p_{1}+\cdots+p_{j}}\right) \\
& \left(x_{S_{H+p_{1}+\cdots+p_{j-1}+1}} \ldots x_{S_{H+p_{1}+\cdots+p_{j}}} \cdot \zeta_{s_{j}^{\prime}} \cdot \eta_{t_{j}^{\prime}}\right) \\
& \wedge \bigwedge_{k=K+1}^{K+L} y_{t_{k}^{\prime}}
\end{aligned}
$$

where, for all $h, a_{1}, \ldots, a_{h} \in \mathbb{N}, R\left(a_{1}, \ldots, a_{h}\right)$ is an integer that appears in the symmetrization map $\otimes^{h} V \rightarrow S^{*}(V)$. We leave the reader to check that indeed

$$
R\left(a_{1}, \ldots, a_{h}\right)=\prod_{i=1}^{n} K\left(i,\left[a_{1}, \ldots, a_{h}\right]\right) ! .
$$

Two indices $i, j \in\{1, \ldots, K+L\}$ are said symmetric with respect to $\mathscr{L}$ if there exists $k \in\{0, \ldots, K-1\}$ such that $i, j \in\left\{\sum_{a=1}^{k} v_{a}, \ldots, \sum_{a=1}^{k+1} v_{a}\right\}$, or if there exists $k \in\left\{0, \ldots, K^{\prime}-1\right\}$ such that $i, j \in\left\{\sum_{a=1}^{k} p_{b}, \ldots, \sum_{a=1}^{k+1} p_{b}\right\}$. Intuitively, $i$ and $j$ are symmetric with respect to $\mathscr{L}$ if the projection $\pi_{\mathscr{L}}$ maps the $i$-th and the $j$-th terms of the tensor product $V^{\otimes(K+L)}$ involved in the definition of $Q$ to the same symmetric algebra $S^{\mu_{a}}(V)$ or to the same symmetric algebra $S^{p_{b}}(V)$.

Two indices $i, j \in\{1, \ldots, K+L\}$ are said antisymmetric with respect to $\mathscr{L}$ if $i \geq K+1$ and $j \geq K+1$. Intuitively, $i$ and $j$ are antisymmetric with respect to $\mathscr{L}$ if the projection $\pi_{\mathscr{L}}$ maps the $i$-th and the $j$-th terms of the tensor product $\left(V^{*}\right)^{\otimes(K+L)}$ involved in the definition of $Q$ to the exterior algebra $\wedge^{L} V^{*}$.

Lemma A.5. Let $\sigma$ be a permutation of $\{1, \ldots, K+L\}$. If there are two indices $i, j \in\{1, \ldots, K+L\}$ symmetric with respect to $\mathscr{L}$ such that $\sigma(i), \sigma(j)$ are antisymmetric with respect to $\mathscr{L}$, then $g_{\sigma, \tau}=0$.

Proof. Consider two indices $i, j \in\{1, \ldots, K+L\}$ symmetric with respect to $\mathscr{L}$ such that $\sigma(i), \sigma(j)$ are antisymmetric with respect to $\mathscr{L}$. For any $k, l \in\{1, \ldots, n\}$, the terms in (A-4) corresponding to $s_{i}=k$ and $s_{j}=l$ and the terms corresponding to $s_{i}=l$ and $s_{j}=k$ appear with opposite signs. By Equation (A-4), $\pi_{\mathscr{L}}\left(g_{\sigma, \tau}\right)$ must therefore vanish.

Lemma A.6. If $\mu_{a} \neq 2$ for some $a \in\{1, \ldots, K\}$, or if $p_{b} \neq 1$ for some $b \in$ $\{1, \ldots, K\}$, then $G_{\mathscr{L}}^{g l_{m} \oplus g l_{m}}=0$.

Proof. If $\mu_{a} \geq 3$ for some $a$ in $\{1, \ldots, K\}$ or if $p_{b} \geq 2$ for some $b$ in $\left\{1, \ldots, K^{\prime}\right\}$, then by (A-3) we must have $L>K+K^{\prime}$. By a simple argument of cardinality, this implies that, for any permutation $\sigma$ of $\{1, \ldots, K+L\}$, there exist $i^{\prime}, j^{\prime} \in$ $\{K+1, \ldots, K+L\}$ such that $\sigma^{-1}\left(i^{\prime}\right), \sigma^{-1}\left(j^{\prime}\right)$ are symmetric with respect to $\mathscr{L}$. Therefore, by Lemma A.5, $\pi_{\mathscr{L}}\left(g_{\sigma, \tau}\right)=0$. 
Assume now that $\mu_{1}=\cdots=\mu_{K}=2$ and $p_{1}=\cdots=p_{K^{\prime}}=1$. In this case,

$$
G_{\mathscr{L}}=\bigwedge^{K}\left(S^{2}(V) \otimes V^{*}\right) \wedge \bigwedge^{K^{\prime}}\left(V \otimes W \otimes W^{*}\right) \wedge \bigwedge^{K+K^{\prime}} V^{*} .
$$

Let $\mathscr{A}$ be the exterior algebra over the space $S^{2}(V) \otimes V^{*} \oplus V \otimes W \otimes W^{*} \oplus V^{*}$. Lemma A.7. If $\sigma$ and $\tau$ are permutations of $\{1, \ldots, K+L\}$ and $\left\{1, \ldots, K^{\prime}\right\}$, respectively, then $\pi_{\mathscr{L}}\left(g_{\sigma, \tau}\right)$ is an element of the algebra generated (with respect to the product $\wedge$ ) by elements of the form

$$
\begin{array}{ll}
\sum_{c=1}^{n} \gamma_{c}^{i} y_{c} \wedge x_{c} x_{i} \cdot y_{j} \in\left(S^{2}(V) \otimes V^{*}\right) \otimes V^{*}, & i, j \in\{1, \ldots, n\}, \\
\sum_{c=1}^{n} y_{c} \wedge x_{c} \cdot \zeta_{k} \cdot \eta_{l} \in\left(V \otimes W \otimes W^{*}\right) \wedge V^{*}, & k, l \in\{1, \ldots, m\},
\end{array}
$$

where, for any $i, c=1, \ldots, n, \gamma_{c}^{i}$ is defined by $\gamma_{c}^{i}=1$ for $i \neq c$ and by $\gamma_{i}^{i}=2$.

Proof. Let $\sigma$ be a permutation of $\left\{1, \ldots, 2 K+K^{\prime}\right\}$. If there is an $i \in\{1, \ldots, K\}$ such that $\sigma(2 i-1) \geq K$ and $\sigma(2 i+1) \geq K$, then Lemma A.5 implies that $\pi_{\mathscr{L}}\left(g_{\sigma, \tau}\right)=$ 0 . Assume now that such an $i \in\{1, \ldots, K\}$ does not exist for $\sigma$; then, for all $i \in\{1, \ldots, K\}$, one of the two integers $\sigma(2 i-1), \sigma(2 i)$ is greater than $K$, and one is smaller or equal to $K$. We define a permutation $\sigma^{\prime}$ of $\{1, \ldots, K\}$ by $\sigma^{\prime}(i)=$ $\min \{\sigma(2 i-1), \sigma(2 i)\}$.

By Lemma A.1(2), we have

$$
\begin{aligned}
\pi_{\mathscr{L}}\left(g_{\sigma, \tau}\right)=\sum_{s_{1}, \ldots, s_{2 K+K^{\prime}}=1}^{n} \sum_{s_{1}^{\prime}, \ldots, s_{K^{\prime}}^{\prime}=1}^{m} \bigwedge_{i=1}^{K} R\left(s_{2 i-1}, s_{2 i}\right)\left(x_{s_{2 i-1}} x_{s_{2 i}} \cdot y_{s_{\sigma(i)}}\right) \\
\wedge \bigwedge_{j=1}^{K^{\prime}} x_{s_{2 K+j}} \zeta_{s_{j}^{\prime}} \cdot \eta_{s_{\tau(j)}^{\prime}} \wedge \bigwedge_{k=K+1}^{2 K+K^{\prime}} y_{s_{\sigma(k)}} .
\end{aligned}
$$

From Equation (A-5), it follows that $R\left(s_{2 i-1}, s_{2 i}\right)=\gamma_{s_{2 i-1}}^{s_{2 i}}$. Therefore,

$$
\begin{aligned}
&\left.\pi_{\mathscr{L}}\left(g_{\sigma, \tau}\right)=\varepsilon \sum_{s_{1}, \ldots, s_{K}=1}^{n} \sum_{s_{1}^{\prime}, \ldots, s_{K^{\prime}}^{\prime}=1}^{m} \bigwedge_{i=1}^{K}\left(\sum_{c=1}^{n} y_{c} \wedge \gamma_{c}^{s_{i}} x_{c} x_{s_{i}} \cdot y_{s_{\sigma^{\prime}(i)}}\right)\right) \\
& \wedge \bigwedge_{j=1}^{K^{\prime}}\left(y_{c} \wedge \sum_{c=1}^{n} x_{c} \cdot \zeta_{t_{i}} \cdot \eta_{t_{\tau(i)}}\right)
\end{aligned}
$$

for some $\varepsilon \in\{-1,+1\}$.

We recapitulate:

Theorem A.8. Let $G_{\mathscr{L}}$ be a $g l_{n} \oplus g l_{m}$-module of type II (as in Definition A.3).

- If $\sum_{a=1}^{K} \mu_{a}+\sum_{b=1}^{K} p_{b} \neq K+L$, then $G_{\mathscr{L}}^{g l_{n} \oplus g l_{m}}=0$.

- If one of the even integers $\left\{v_{a}\right\}_{a=1, \ldots, K}$ is not 0 , then $G_{\mathscr{L}}^{g l_{n} \oplus g l_{m}}=0$.

- If one of the odd integers $\left\{q_{b}\right\}_{b=1, \ldots, K^{\prime}}$ is not 1 , then $G_{\mathscr{L}}^{g l_{n} \oplus g l_{m}}=0$.

- If one of the integers $\left\{\mu_{a}\right\}_{a=1, \ldots, K}$ is not 2 , then $G_{\mathscr{L}}^{g l_{n} \oplus g l_{m}}=0$. 
- If one of the integers $\left\{p_{b}\right\}_{b=1, \ldots, K^{\prime}}$ is not 1 , then $G_{\mathscr{L}}^{g l_{n} \oplus g l_{m}}=0$.

- If $\mu_{a}=2$ and $v_{a}=0$ for $a=1, \ldots, n$, and if $p_{b}=q_{b}=1$ for $b=1, \ldots, m$, then the space $G_{\mathscr{L}}^{g l_{n} \oplus g l_{m}}$ is contained in the subalgebra of $A$ generated by the elements

$$
\begin{cases}\sum_{c=1}^{n} y_{c} \wedge\left(\gamma_{c}^{i} x_{c} \cdot x_{i} \cdot y_{j}\right), & i, j \in\{1, \ldots, n\} \\ \sum_{c=1}^{n} y_{c} \wedge x_{c} \cdot \zeta_{k} \cdot \eta_{l}, & k, l \in\{1, \ldots, m\} .\end{cases}
$$

Later, we will also need the following:

Lemma A.9 [Fuchs 1986]. For any $g l_{n} \oplus g l_{m}$-module $E$ of finite dimension,

$$
H^{*}\left(g l_{n} \oplus g l_{m}, E\right)=H^{*}\left(g l_{n} \oplus g l_{m}, E^{g l_{n} \oplus g l_{m}}\right) .
$$

Actually, $H^{*}\left(g l_{n} \oplus g l_{m}, E\right)=H^{*}\left(g l_{n} \oplus g l_{m}, E^{g l_{n} \oplus g l_{m}}\right)=H^{*}\left(g l_{n} \oplus g l_{m}, \mathbb{R}\right) \otimes$ $E^{g l_{n} \oplus g l_{m}}$.

A2. The cohomology of $\operatorname{Vect}(n, m)_{0}$ and the Weil algebra. Now we are able to prove Theorem 3.7. Let $\mathscr{K}$ be the kernel of the DGA homomorphism $\tilde{\alpha}: W\left(g l_{n} \oplus\right.$ $\left.g l_{m}\right) \rightarrow \bigwedge \operatorname{Vect}(n, m)_{0}^{*}$, and denote again by $\tilde{\alpha}$ the induced DGA homomorphism from $W\left(g l_{n} \oplus g l_{m}\right) / \mathscr{K}$ to $\bigwedge \operatorname{Vect}(n, m)_{0}^{*}$. We restate the theorem:

Theorem 3.7. Let $\hat{\alpha}$ be the map from $H^{*}\left(W\left(g l_{n} \oplus g l_{m}\right) / \mathscr{K}\right)$ to $H^{*}\left(\operatorname{Vect}(n, m)_{0}\right)$ induced by $\tilde{\alpha}$. The map $\hat{\alpha}$ is an isomorphism.

Proof. We first describe $\tilde{\alpha}: W\left(g l_{n} \oplus g l_{m}\right) \rightarrow \bigwedge \operatorname{Vect}(n, m)_{0}^{*}$ precisely. By (3-12), the DGA homomorphism $\tilde{\alpha}$ from $W\left(g l_{n} \oplus g l_{m}\right)$ to $\wedge \operatorname{Vect}(n, m)_{0}^{*}$ is given by:

$$
1 \otimes a_{i, j} \mapsto\left(x_{i} \partial / \partial x_{j}\right)^{*}, \quad 1 \otimes d_{i, j} \mapsto\left(\theta_{i} \partial / \partial \theta_{j}\right)^{*},
$$

where $a_{i, j}$, for $i, j \in\{1, \ldots, n\}$, and $d_{i, j}$, for $i, j \in\{1, \ldots, m\}$, are bases that are dual to the canonical bases of $g l_{n}$ and $g l_{m}$, respectively.

One can easily check that

$$
\left\{\begin{array}{l}
\partial\left(x_{i} \frac{\partial}{\partial x_{j}}\right)^{*}-\sum_{k=1}^{n}\left(x_{i} \frac{\partial}{\partial x_{k}}\right)^{*} \wedge\left(x_{k} \frac{\partial}{\partial x_{j}}\right)^{*}=\sum_{c=1}^{n}\left(\frac{\partial}{\partial x_{c}}\right)^{*} \wedge\left(\frac{x_{c} x_{i}}{\gamma_{c}^{i}} \frac{\partial}{\partial x_{j}}\right)^{*}, \\
\partial\left(\theta_{i} \frac{\partial}{\partial \theta_{j}}\right)^{*}-\sum_{k=1}^{m}\left(\theta_{i} \frac{\partial}{\partial \theta_{k}}\right)^{*} \wedge\left(\theta_{k} \frac{\partial}{\partial \theta_{j}}\right)^{*}=\sum_{c=1}^{n}\left(\frac{\partial}{\partial x_{c}}\right)^{*} \wedge\left(x_{c} \theta_{i} \frac{\partial}{\partial \theta_{j}}\right)^{*} .
\end{array}\right.
$$

Since $\tilde{\alpha}$ is a DGA homomorphism, by the definition of the differential of a Weil algebra [Guillemin and Sternberg 1999], we have

$$
\begin{aligned}
& \tilde{\alpha}\left(a_{i, j} \otimes 1\right)=\sum_{c=1}^{n}\left(\frac{\partial}{\partial x_{c}}\right)^{*} \wedge\left(\frac{x_{c} x_{i}}{\gamma_{c}^{i}} \frac{\partial}{\partial x_{j}}\right)^{*}, \\
& \tilde{\alpha}\left(d_{i, j} \otimes 1\right)=\sum_{c=1}^{n}\left(\frac{\partial}{\partial x_{c}}\right)^{*} \wedge\left(x_{c} \theta_{i} \frac{\partial}{\partial \theta_{j}}\right)^{*} .
\end{aligned}
$$


We compute the cohomology of $\operatorname{Vect}(n, m)_{0}$ with the help of the HochschildSerre spectral sequence [Fuchs 1986] associated to the sub-Lie algebra of elements of weight zero. Its second term $E_{i, j}^{2}$ is

$$
E_{i, j}^{2}=H^{i}\left(g l_{n} \oplus g l_{m}, \bigwedge^{j} \bigoplus_{k \neq 0} F_{k}\right)
$$

where $F_{k}$ is the space of elements of weight $k$ in $\left(\operatorname{Vect}(n, m)_{0}\right)^{*}$. By Lemma A.9, $E_{i, j}^{2}$ reduces to

$$
E_{i, j}^{2}=H^{i}\left(g l_{n} \oplus g l_{m},\left(\bigwedge^{j} \bigoplus_{k \neq 0} F_{k}\right)^{g l(n) \oplus g l_{m}}\right) .
$$

The $g l_{n} \oplus g l_{m}$-module $\bigwedge^{j} \bigoplus_{k \neq 0} F_{k}$ is a direct sum of modules of type II. For example,

$$
F_{-1} \simeq W^{*} \quad \text { and } \quad F_{1}=\left(S^{2}(V) \otimes V^{*}\right) \oplus\left(V \otimes W \otimes W^{*}\right) .
$$

More generally, denoting by $\lfloor x\rfloor$ the integer part of $x \in \mathbb{R}$,

$$
F_{k} \simeq \bigoplus_{i=0}^{\lfloor(k+1) / 2\rfloor}\left(V^{\otimes k+1-2 i} \otimes W^{\otimes 2 i} \otimes V^{*}\right) \oplus \bigoplus_{j=0}^{\lfloor k / 2\rfloor}\left(V^{\otimes k+1-(2 j+1)} \otimes W^{\otimes(2 j+1)} \otimes W^{*}\right) .
$$

Therefore, the modules $\wedge^{j_{1}} F_{i_{1}} \wedge \cdots \wedge \wedge^{j_{k}} F_{i_{k}}$, where $i_{1}, \ldots, i_{k} \in\{-1,1,2,3, \ldots\}$ and $j_{1}, \ldots, j_{k} \in \mathbb{N}$, are direct sums of modules of type II.

The $g l_{n} \oplus g l_{m}$-module

$$
\bigwedge^{j} F_{-1} \otimes \bigwedge^{j} F_{1}
$$

is a module of type II with $\mu_{a}=2$ and $v_{a}=0$ for $a=1, \ldots, j$, with $p_{b}=1$ and $q_{b}=1$ for $b=1, \ldots, j$, and with $L=j$. By Theorem A.8, the modules $\bigwedge^{j_{1}} F_{i_{1}} \wedge \cdots \wedge \wedge^{j_{k}} F_{i_{k}}$ have no nontrivial space of invariants except those of the form $\wedge^{j} F_{-1} \wedge \wedge^{j} F_{1}$. Therefore,

$$
\left\{\begin{array}{l}
\left(\bigwedge^{2 j} \bigoplus_{k \neq 0} F_{k}\right)^{g l_{n} \oplus g l_{m}}=\left(\bigwedge^{j} F_{-1} \otimes \bigwedge^{j} F_{1}\right)^{g l_{n} \oplus g l_{m}}, \\
\left(\bigwedge^{2 j+1} \bigoplus_{k \neq 0} F_{k}\right)^{g l_{n} \oplus g l_{m}}=0 .
\end{array}\right.
$$

By Theorem A.8(6), we obtain that the $g l_{n} \oplus g l_{m}$-invariant elements of $\bigwedge^{j} \bigoplus_{k \neq 0} F_{k}$ are elements of the subalgebra of $\bigwedge \operatorname{Vect}(n, m)_{0}^{*}$ generated by

$$
\begin{cases}\sum_{c=1}^{n} \gamma_{c}^{i}\left(\frac{\partial}{\partial x_{c}}\right)^{*} \wedge\left(x_{c} x_{i} \frac{\partial}{\partial x_{j}}\right)^{*}, & i, j \in\{1, \ldots, n\}, \\ \sum_{c=1}^{m}\left(\frac{\partial}{\partial x_{c}}\right)^{*} \wedge\left(x_{c} \theta_{k} \frac{\partial}{\partial \theta_{l}}\right)^{*}, & k, l \in\{1, \ldots, m\} .\end{cases}
$$


From (3-3), the identity $\gamma_{c}^{i}\left(x_{c} x_{i} \partial / \partial x_{j}\right)^{*}=\left(\left(x_{c} x_{i} / \gamma_{c}^{i}\right) \partial / \partial x_{j}\right)^{*}$ holds. Hence, the generators can be written

$$
\left\{\begin{array}{l}
\sum_{c=1}^{n}\left(\frac{\partial}{\partial x_{c}}\right)^{*} \wedge\left(\frac{x_{c} x_{i}}{\gamma_{c}^{i}} \frac{\partial}{\partial x_{j}}\right)^{*}, \quad i, j \in\{1, \ldots, n\}, \\
\sum_{c=1}^{m}\left(\frac{\partial}{\partial x_{c}}\right)^{*} \wedge\left(x_{c} \theta_{k} \frac{\partial}{\partial \theta_{l}}\right)^{*}, \quad k, l \in\{1, \ldots, m\} .
\end{array}\right.
$$

Let $\mathscr{I}=\tilde{\alpha}\left(W\left(g l_{n} \oplus g l_{m}\right)\right)$ be the sub-DGA of $\bigwedge^{*}\left(\operatorname{Vect}(n, m)_{0}\right)$ that is the image of the complex $W\left(g l_{n} \oplus g l_{m}\right)$ through $\tilde{\alpha}$. By (A-8) and (A-9), we have

$$
\left(\bigwedge^{j} \bigoplus_{k \neq 0} F_{k}\right)^{g l_{n} \oplus g l_{m}} \subset \Phi .
$$

Moreover, by Equation (A-6) we have the identity

$$
\mathscr{I} \cap F_{0}=F_{0} \simeq g l_{n} \oplus g l_{m} .
$$

The cohomology of $\mathscr{I}$ can be computed with the help of a spectral sequence whose second term is

$$
\tilde{E}_{i, j}^{2}=H^{i}\left(I \cap F_{0}, I \cap\left(\bigwedge^{j} \bigoplus_{k \neq 0} F_{k}\right)\right) .
$$

By (A-11), $\tilde{E}_{i, j}^{2}=H^{i}\left(g l_{n} \oplus g l_{m}, \quad \Phi \cap\left(\bigwedge^{j} \bigoplus_{k \neq 0} F_{k}\right)\right)$. From Lemma A.9 we

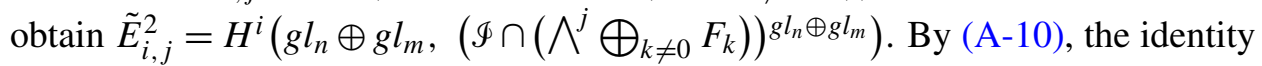
$\tilde{E}_{i, j}^{2}=E_{i, j}^{2}$ holds.

Therefore, the cohomology of $\bigwedge \operatorname{Vect}(n, m)_{0}^{*}$ is equal to the cohomology of the subcomplex $\mathscr{I}=\tilde{\alpha}\left(W\left(g l_{n} \oplus g l_{m}\right)\right) \simeq W\left(g l_{n} \oplus g l_{m}\right) / \mathscr{K}$. This completes the proof of Theorem 3.7.

As an application, we compute the cohomology of $H^{*}\left(\operatorname{Vect}(n, m)_{0}\right)$ in some particular cases:

Proposition A.10. The cohomology of $\operatorname{Vect}(0, m)_{0}$ is isomorphic to the ChevalleyEilenberg cohomology of the Lie algebra $\mathrm{gl}_{\mathrm{m}}$.

Proof. The DGA homomorphism from $W\left(g l_{m}\right)$ to $\bigwedge \operatorname{Vect}(0, m)_{0}^{*}$ is:

$$
1 \otimes d_{i, j} \mapsto\left(\theta_{i} \partial / \partial \theta_{j}\right)^{*} \quad \text { and } \quad d_{i, j} \otimes 1 \mapsto 0 .
$$

The kernel of this homomorphism is $S(g l(m)) \otimes 1$. The cohomology of

$$
W\left(g l_{m}\right) / S(g l(m)) \otimes 1=\bigwedge g l_{m}
$$

is the Chevalley-Eilenberg cohomology of the Lie algebra $g l_{m}$.

In particular: 
Proposition A.11. We have isomorphisms $H^{1}\left(\operatorname{Vect}(0,1)_{0}\right) \simeq H^{0}\left(\operatorname{Vect}(0,1)_{0}\right) \simeq$ $\mathbb{R}$, and $H^{1}\left(\operatorname{Vect}(0,1)_{0}\right)=0$. Moreover, $H^{1}\left(\operatorname{Vect}(0,1)_{0}\right)$ is generated by $(\theta \partial / \partial \theta)^{*}$.

We also determine the cohomology of $\operatorname{Vect}(1,1)_{0}$ :

Proposition A.12. The cohomology of $\operatorname{Vect}(1,1)_{0}$ is given by $H^{n}\left(\operatorname{Vect}(1,1)_{0}\right)=0$ if $n \neq 0,3$, and by $H^{0}\left(\operatorname{Vect}(1,1)_{0}\right)=\mathbb{R}$ and $H^{3}\left(\operatorname{Vect}(1,1)_{0}\right)=\mathbb{R}^{3}$. Moreover, the latter is generated by the classes described in (A-13), (A-14), and (A-15).

Proof. We denote by $a$ and $d$ the generators $a_{1,1}$ and $d_{1,1}$ of $g l_{1} \oplus g l_{1}$. The DGA homomorphism from $W\left(g l_{1} \oplus g l_{1}\right)$ to $\bigwedge \operatorname{Vect}(1,1)_{0}^{*}$ is given by

$(\mathrm{A}-12)$

$$
\left\{\begin{array}{l}
1 \otimes a \mapsto\left(x \frac{\partial}{\partial x}\right)^{*}, \\
1 \otimes d \mapsto\left(\theta \frac{\partial}{\partial \theta}\right)^{*}, \\
a \otimes 1 \mapsto 2\left(\frac{\partial}{\partial x}\right)^{*} \wedge\left(x^{2} \frac{\partial}{\partial x}\right)^{*}=\left(\frac{\partial}{\partial x}\right)^{*} \wedge\left(\frac{x^{2}}{2} \frac{\partial}{\partial x}\right)^{*}, \\
d \otimes 1 \mapsto\left(\frac{\partial}{\partial x}\right)^{*} \wedge\left(x \theta \frac{\partial}{\partial \theta}\right)^{*} .
\end{array}\right.
$$

The kernel $\mathscr{K}$ of this application is generated by $S^{2}\left(g l_{1} \oplus g l_{1}\right) \otimes 1$. This implies that the cohomology vanishes in all degrees different from 3 . We can now compute the cohomology of $W\left(g l_{1} \oplus g l_{1}\right) / \mathscr{K}$. It is easy to check that $H^{3}\left(\operatorname{Vect}(1,1)_{0}\right)=\mathbb{R}^{3}$. Generators of $H^{3}\left(\operatorname{Vect}(1,1)_{0}\right)$ are given by

$$
\begin{aligned}
& H_{1}=\left[\left(\frac{x^{2}}{2} \frac{\partial}{\partial x}\right)^{*} \wedge\left(x \frac{\partial}{\partial x}\right)^{*} \wedge\left(\frac{\partial}{\partial x}\right)^{*}\right], \\
& H_{2}=\left[\left(x \theta \frac{\partial}{\partial \theta}\right)^{*} \wedge\left(x \frac{\partial}{\partial x}\right)^{*} \wedge\left(\frac{\partial}{\partial x}\right)^{*}\right], \\
& H_{3}=\left[\left(x \theta \frac{\partial}{\partial \theta}\right)^{*} \wedge\left(\theta \frac{\partial}{\partial \theta}\right)^{*} \wedge\left(\frac{\partial}{\partial x}\right)^{*}\right] .
\end{aligned}
$$

\section{Appendix B. Proof of Lemma 4.3}

Lemma 4.3. Let $d_{1}, \ldots, d_{n}$ be odd 1 -forms and $d_{n+1}, \ldots, d_{n+m}$ even 1-forms, forming a free family. If $d^{i}, i \in\{1, \ldots, n+m\}$, are 2-forms on $M$ such that, for any $j \in\{1, \ldots, n+m\}$,

$$
\sum_{i=1}^{n+m} d_{i} \wedge d^{i}=0
$$

then there exist homogeneous 1 -forms $d^{i, j}$, of parity $|i|+|l|+1$, with

$$
d^{i, l}=-(-1)^{(|i|+1)(|l|+1)} d^{l, i} \quad \text { and } \quad \sum_{i=1}^{n+m} d_{i} \wedge d^{i, l}=d^{l} .
$$


Proof. There are partitions of unity on $\mathcal{M}$, that is, for any open covering $\left\{U_{s}\right\}_{s \in S}$ of the base manifold, there exist even superfunctions $\left\{\varphi_{s} \in \Omega(\mathcal{M})\right\}_{s \in S}$ with support in $U_{s}$ and such that $\sum_{s \in S} \varphi_{s}=1_{\mathcal{M}}$, where $1_{\mathcal{M}}$ is the unit of $\mathcal{O}(\mathcal{M})$. As consequence, if there is an open covering $\left\{U_{s}\right\}_{s \in S}$, of the base manifold such that (B-2) has local solutions on $U_{s}$ for any $s \in S$, then (B-2) has a global solution. We therefore only have to prove that (B-2) has solutions locally.

For this, consider 1-forms $d_{n+m+1}, \ldots, d_{p+q}$ such that $d_{1}, \ldots, d_{p+q}$ is a trivialization of $\Omega^{1}(\mathcal{M}) \simeq T \mathcal{M}^{*}$. For any $i \in\{1, \ldots, p+q\}$, define $|i|$ to be 1 if $d_{i}$ is even, and 0 otherwise. (This definition generalizes the previous definition of $|i|$ given in Definition 3.3.)

For any $l \in\{1, \ldots, n+m\}$, there exist superfunctions $F^{r, s ; i}, G^{t, u ; i}$, and $H^{v, w ; i}$, with $r, s, t \in\{1, \ldots, n+m\}, u, v, w \in\{n+m+1, \ldots, p+q\}, i \in\{1, \ldots, n+m\}$, such that

$$
\left\{\begin{array}{l}
d^{l}=\sum_{r, s=1}^{n+m} d_{r} \wedge d_{s} \wedge F^{r, s ; l}+\sum_{t=1}^{n+m} \sum_{u=n+m+1}^{p+q} d_{t} \wedge d_{u} \wedge G^{t, u ; l} \\
\quad+\sum_{v, w=n+m+1}^{p+q} d_{v} \wedge d_{w} \wedge H^{v, w ; l}, \\
F^{r, s ; l}=(-1)^{(|r|+1)(|s|+1)} F^{s, r ; l}, \\
H^{v, w ; l}=(-1)^{(|v|+1)(|w|+1)} H^{w, v ; l} .
\end{array}\right.
$$

For convenience, we have chosen to multiply a 1-form with a superfunction on the right, which is unusual but will simplify the signs. Equation (B-1) gives

$$
\begin{aligned}
\sum_{l=1}^{n+m} \sum_{r, s=1}^{n+m} d_{l} \wedge d_{r} \wedge d_{s} \wedge F^{r, s ; l}+\sum_{l=1}^{n+m} \sum_{t=1}^{n+m} \sum_{u=n+m+1}^{p+q} d_{l} \wedge d_{t} \wedge d_{u} \wedge G^{t, u ; l} & \\
& +\sum_{l=1}^{n+m} \sum_{v, w=n+m+1}^{p+q} d_{l} \wedge d_{v} \wedge d_{w} \wedge H^{v, w ; l}=0
\end{aligned}
$$

This is equivalent to the three conditions

$$
\left\{\begin{array}{l}
F^{r, s ; l}+(-1)^{(|l|+1)(|s|+|r|)} F^{s, l ; r}+(-1)^{(|s|+1)(|r|+|l|)} F^{l, r ; s}=0 \\
G^{t, u ; l}=-(-1)^{(|l|+1)(|t|+1)} G^{l, u ; t} \\
H^{v, w ; l}=0
\end{array}\right.
$$

Define $\left(d^{i ; l}\right)_{i, l=1, \ldots, n+m}$ by

$$
\begin{aligned}
d^{i ; l}=\frac{4}{3} \sum_{s=1}^{n+m} d_{s} \wedge\left(F^{i, s ; l}+\frac{1}{2}(-1)^{|s|(|i|+1)+|i|(|l|+1)} d_{s}\right. & \left.\wedge F^{i, l ; s}\right) \\
& +\sum_{u=n+m+1}^{p+q} d_{u} \wedge G^{i, u ; l} .
\end{aligned}
$$

We check that (B-4) implies that (B-2) can be satisfied: 
Step 1: Checking that $d^{i ; l}=(-1)^{(|i|+1)(|l|+1)} d^{l ; i}$.

From $G^{t, u ; i}=(-1)^{(|i|+1)(|l|+1)} G^{i, u ; l}$, we obtain

$$
\sum_{u=n+m+1}^{p+q} d_{u} \wedge G^{i, u ; l}=(-1)^{(|i|+1)(|l|+1)} \sum_{u=n+m+1}^{p+q} d_{t} \wedge G^{l, u ; i} .
$$

Moreover, from (B-3) and (B-4), we obtain

$$
F^{l, s ; i}+(-1)^{(|s|+1)(|l|+|i|)} F^{i, l ; s}+(-1)^{(|l|+1)(|i|+1)} F^{i, s ; l}=0 .
$$

It is then straightforward to check that

$$
\begin{aligned}
F^{l, s ; i}+\frac{1}{2}(-1)^{(|s|+1)(|l|+|i|)} & F^{l, i ; s} \\
& =-(-1)^{(|l|+1)(|i|+1)}\left(F^{i, s ; l}+\frac{1}{2}(-1)^{(|s|+1)(|l|+|i|)} F^{i, l ; s}\right) .
\end{aligned}
$$

This and (B-6) imply that

$$
d^{i ; l}=-(-1)^{(|i|+1)(|l|+1)} d^{l ; i} .
$$

Step 2: Checking that $\sum_{i=1}^{n+m} d_{i} \wedge d^{i ; l}=d^{l}$.

We compute $\sum_{i, s=1}^{n+m}(-1)^{(|s|+1)(|l|+|i|)} d_{i} \wedge d_{s} F^{i, l ; s}$. It is equal to

$$
\frac{1}{2} \sum_{i, s=1}^{n+m}(-1)^{(|s|+1)(|l|+|i|)} d_{i} \wedge d_{s} F^{i, l ; s}+\frac{1}{2} \sum_{i, s=1}^{n+m}(-1)^{(|s|+1)(|l|+|i|)} d_{s} \wedge d_{i} \wedge F^{s, l ; i}
$$

From the identity $(-1)^{(|s|+1)(|l|+|i|)}(-1)^{(|s|+1)(|i|+1)}=(-1)^{(|l|+1)(|s|+1)}$, we deduce

$$
\begin{aligned}
\sum_{i, s=1}^{n+m}(-1)^{|s|(|i|+1)+|i|(|l|+1)} d_{i} & \wedge d_{s} \wedge F^{i, l ; s} \\
= & \frac{1}{2} d_{i} \wedge d_{s} \wedge\left((-1)^{(|s|+1)(|l|+|i|)} F^{i, l ; s}+\frac{1}{2}(-1)^{(|l|+1)(|s|+1)} F^{s, l ; i}\right) \\
= & \frac{1}{2} d_{i} \wedge d_{s} \wedge\left((-1)^{(|s|+1)(|l|+|i|)} F^{i, l ; s}+(-1)^{(|l|+1)(|i|+1)} F^{l, s ; i}\right) .
\end{aligned}
$$

From (B-7), we obtain

$$
\sum_{i, s=1}^{n+m}(-1)^{(|s|+1)(|l|+|i|)} d_{i} \wedge d_{s} F^{i, l ; s}=-\frac{1}{4} \sum_{i, s=1}^{n+m} d_{i} \wedge d_{s} \wedge F^{i, s ; l}
$$

The result now follows immediately from (B-5) and (B-9).

\section{References}

[Astashkevich and Fuchs 1993] A. B. Astashkevich and D. B. Fuchs, "On the cohomology of the Lie superalgebra $W(m \mid n)$ ", pp. 1-13 in Unconventional Lie algebras, edited by D. Fuchs, Adv. Soviet Math. 17, Amer. Math. Soc., Providence, RI, 1993. MR 94k:17003 Zbl 0801.17021

[Bernstein and Rosenfeld 1973] I. N. Bernšteřn and B. I. Rosenfel'd, "Homogeneous spaces of infinite-dimensional Lie algebras and the characteristic classes of foliations", Uspehi Mat. Nauk 28:4(172) (1973), 103-138. MR 54 \#3714 Zbl 0289.57011 
[Bott and Haefliger 1972] R. Bott and A. Haefliger, "On characteristic classes of $\Gamma$-foliations", Bull. Amer. Math. Soc. 78 (1972), 1039-1044. MR 46 \#6370 Zbl 0262.57010

[Fuchs 1973] D. B. Fuchs, "Characteristic classes of foliations”, Uspehi Mat. Nauk 28:2(170) (1973), 3-17. MR 54 \#3716 Zbl 0274.57005

[Fuchs 1986] D. B. Fuchs, Cohomology of infinite-dimensional Lie algebras, Contemporary Soviet Mathematics, Consultants Bureau, New York, 1986. MR 88b:17001 Zbl 0667.17005

[Godbillon and Vey 1971] C. Godbillon and J. Vey, "Un invariant des feuilletages de codimension 1”, C. R. Acad. Sci. Paris Sér. A-B 273 (1971), A92-A95. MR 44 \#1046 Zbl 0215.24604

[Guillemin and Sternberg 1999] V. W. Guillemin and S. Sternberg, Supersymmetry and equivariant de Rham theory, Mathematics Past and Present, Springer, Berlin, 1999. MR 2001i:53140 Zbl 0934.55007

[Hill and Simanca 1991] C. D. Hill and S. R. Simanca, "The super complex Frobenius theorem", Ann. Polon. Math. 55 (1991), 139-155. MR 93e:32009 Zbl 0756.5800

[Howe 1989] R. Howe, "Remarks on classical invariant theory", Trans. Amer. Math. Soc. 313:2 (1989), 539-570. MR 90h:22015a Zbl 0674.15021

[Kamber and Tondeur 1974] F. W. Kamber and P. Tondeur, "Characteristic invariants of foliated bundles”, Manuscripta Math. 11 (1974), 51-89. MR 48 \#12556 Zbl 0267.57012

[Kamber and Tondeur 1975] F. W. Kamber and P. Tondeur, Foliated bundles and characteristic classes, Springer, Berlin, 1975. MR 53 \#6587 Zbl 0308.57011

[Koszul 1988] J.-L. Koszul, "Les superalgèbres de Lie W(n) et leurs représentations", pp. 161-171 in Géométrie différentielle (Paris, 1986), edited by D. Bernard and Y. Choquet-Bruhat, Travaux en Cours 33, Hermann, Paris, 1988. MR 90d:17006 Zbl 0652.17014

[Laurent-Gengoux 2004] C. Laurent-Gengoux, "Secondary characteristic classes of superfoliations", C. R. Math. Acad. Sci. Paris 339:8 (2004), 567-572. MR 2005i:58006 Zbl 1065.58004

[Leites 1980] D. A. Ley̆tes, "Introduction to the theory of supermanifolds", Uspekhi Mat. Nauk 35:1(211) (1980), 3-57, 255. MR 81j:58003 Zbl 0462.58002

[Monterde et al. 1997] J. Monterde, J. Muñoz-Masqué, and O. A. Sánchez-Valenzuela, "Geometric properties of involutive distributions on graded manifolds", Indag. Math. (N.S.) 8:2 (1997), 217246. MR 99c:58007 Zbl 0887.58008

[Tuynman 2004] G. M. Tuynman, Supermanifolds and supergroups: basic theory, Mathematics and its Applications 570, Kluwer Academic Publ., Dordrecht, 2004. MR 2005k:58007 Zbl 1083.58001

Received February 3, 2005.

\section{CAMille Laurent-GengouX}

DÉPARTEMENT DE MATHÉMATIQUES

UNIVERSITÉ DE POITIERS

TÉléport 2, Boulevard Marie et Pierre Curie BP 30179

86962 Futuroscope Chasseneuil CedeX

FRANCE

laurent@math.univ-poitiers.fr 